

\title{
Modeling Scalar Dissipation And Scalar Variance In LES: Algebraic And Transport Equation Closures
}

\author{
E. Knudsen ${ }^{\mathrm{a}, *}$, E. S. Richardson ${ }^{\mathrm{b}}$, E. M. Doran ${ }^{\mathrm{c}}$, H. Pitsch ${ }^{\mathrm{d}}$, J. H. Chen ${ }^{\mathrm{e}}$ \\ ${ }^{a}$ Dept. of Mechanical Engineering, Stanford University, Stanford, California 94305, USA \\ ${ }^{b}$ Faculty of Engineering and the Environment, University of Southampton, SO17 1BJ, UK \\ ${ }^{c}$ Robert Bosch RTC, Palo Alto, California 94304, USA \\ ${ }^{d}$ Institut für Technische Verbrennung, Templergraben 64, 52056 Aachen, Germany \\ ${ }^{e}$ Sandia National Lab, Combustion Research Facility, PO Box 969, Livermore, CA 94551, USA
}

\begin{abstract}
The scalar dissipation rate and the scalar variance are important modeling parameters in large eddy simulations (LES) of reacting flows. Approaches ranging from constant coefficient algebraic equations to transport equations with complex closure models have been proposed to describe these parameters. While currently available approaches capture many of the physics behind the dissipation and variance, their modeling remains challenging. Here, two direct numerical simulations (DNS) are used to analyze LES dissipation rate and variance models, and to propose a new model for the dissipation rate that is based on a transport equation. The first DNS that is considered is a non-premixed auto-igniting $\mathrm{C}_{2} \mathrm{H}_{4}$ jet flame simulation performed by Yoo et al. (Proc. Comb. Inst., 2010). An LES of this case is run using algebraic models for the dissipation rate and variance. It is shown that the algebraic models do not accurately reproduce the subfilter DNS fields. This motivates the introduction of a transport equation model for the LES dissipation rate. Closure of the equation is addressed by formulating a new adapted dynamic approach. This approach borrows dynamically computed information from LES quantities that, unlike the dissipation rate, do not reside on the smallest flow length scales. The adapted dynamic closure is analyzed by considering a second DNS of scalar mixing in homogeneous isotropic turbulence. Data from this second DNS is used to confirm that the adapted dynamic procedure successfully closes the dissipation rate equation over a wide range of LES filter widths. The first reacting jet case is then returned to and used to test the LES transport equation models. These models are shown to predict the dissipation rate and variance fields more accurately than the the algebraic LES models.
\end{abstract}

Keywords: Large Eddy Simulation, Scalar Dissipation Rate, Scalar Variance, Subfilter Closure Modeling, Combustion Modeling

$P A C S$ :

\section{Introduction}

Scalar dissipation rates and subfilter scalar variances are critical modeling parameters in large eddy simulations (LES) of reacting flows. In some reacting flow models such as the flamelet approach $[1,2,3]$ and the conditional moment closure approach $[4,5]$, these parameters are explicitly used to calculate filtered quantities. In other reacting flow models

\footnotetext{
* Corresponding author.

Email address: ewk@stanford.edu (E. Knudsen)
} 
such as transported density function approaches [6], the importance of the variance and dissipation rate is embedded in model variables such as mixing coefficients [7]. - A variety of models that attempt to describe the scalar dissipation rate and scalar variance have been proposed. Many have been designed to operate within a Reynolds averaged framework, and have been subsequently adopted for use in LES. Dissipation rate and variance models can be categorized according to whether they are based on an algebraic equation or a transport equation. Algebraic models for the variance $[8,9,10,11,12]$ and the dissipation rate $[8,13$, 14] directly describe the modeled parameter using information about the local scalar field and filter width, and have the advantage of being conceptually simple and computationally inexpensive. Transport equation models for the variance $[12,15,16]$ and for the dissipation rate $[17,18,19]$ offer different advantages: they do not forcibly assume that production and dissipation processes are in equilibrium, they produce fields that are less noisy than algebraic models, and they incorporate a wider range of physics. Nonetheless, transport equation approaches are sensitive to the descriptions used to model unclosed subfilter terms, and the performance of these equations is not always superior to the performance of algebraic models [12]. For example, the closure of these transport equations may be strongly affected by the details and accuracy of the numerical method that is used. Consequently, many uncertainties continue to surround the modeling of scalar variance and dissipation.

These uncertainties are important because reacting LES results have been proven sensitive to the scalar variance and dissipation rate. For example, Domingo et al. [16] tested two forms of an algebraic model for the progress variable dissipation rate in an LES of a lifted jet. It was observed that changing the form of the subfilter dissipation rate closure led to significant changes in the predictions of minor species such as CO. Ihme et al. [20], considering the same case, demonstrated that the flame's lift-off height would change as a function of the model assumptions used in the calculation of the subfilter progress variable variance. Finally, Pitsch [21] found that improved descriptions of the mixture fraction dissipation rate in an LES of a steady non-premixed flame led to improved predictions of several minor species. Even in the context of a rather stable flame, sensitivities to the modeling of local scalar dissipation could be demonstrated.

Two observations can be extracted from these examples. First, improvements in variance and dissipation rate LES models are expected to significantly improve the quality of reactive flow predictions. Second, significant ambiguity exists regarding how these improvements should be pursued. The goal of this work is to further address these modeling needs by making use of newly available reacting direct numerical simulation data, and by proposing a new LES closure model. The discussion will proceed as follows. This introduction forms section 1 of the paper, and in section 2 baseline models for the scalar dissipation rate and scalar variance are reviewed. A reacting DNS case is introduced in section 3. The performance of LES subfilter algebraic models is described and discussed in section 4 . In section 5, a new closure for the dissipation rate transport equation is formally developed. This development process introduces a second DNS of scalar mixing in homogeneous turbulence. Finally, the new closure model is applied in additional simulations of the reacting jet in section 6 . Conclusions and a summary are provided in section 7 .

\section{Modeling Background}

The scalar dissipation rate $\chi_{Z}$ of a scalar $Z$ whose diffusivity is $\mathcal{D}_{Z}$ can be written

$$
\chi_{Z}=2 \mathcal{D}_{Z}|\nabla Z|^{2} .
$$

When an LES filter is applied to this quantity, the filtered dissipation rate is found,

$$
\bar{\chi}_{Z}=2 \overline{\mathcal{D}_{Z}|\nabla Z|^{2}},
$$


where the $\left({ }^{-}\right)$operator denotes spatial filtering. The subfilter scalar variance is typically calculated using density weighted filtering that will be denoted by the tilde operator $(\widetilde{\sim})$. This subfilter variance is

$$
\widetilde{Z_{s f s}^{\prime \prime 2}}=\widetilde{Z^{2}}-(\widetilde{Z})^{2}
$$

\subsection{Algebraic Models}

Algebraic LES models for $\bar{\chi}_{Z}$ can be formulated using a variety of parameters [14]. The simplest and most widely used formulation separates the dissipation rate into resolved and subfilter components [13],

$$
\bar{\chi}_{Z}=2\left(\overline{\mathcal{D}}_{Z}+\mathcal{D}_{t}\right)|\nabla \widetilde{Z}|^{2},
$$

where the filtered molecular diffusivity $\overline{\mathcal{D}}_{Z}$ is associated with the resolved dissipation, and the turbulent diffusivity $\mathcal{D}_{t}$ is associated with the subfilter dissipation. This model introduces no unknown coefficients, as the turbulent diffusivity is already available from the solution of the underlying scalar's transport equation. In all calculations shown here, $\mathcal{D}_{t}$ is calculated by applying a dynamic Smagorinsky model to the relevant scalar and then using Lagrangian averaging [22].

Algebraic LES models for the subfilter scalar variance can also take many forms $[9,10$, 11]. The most widely used form relates the variance to gradients of the resolved scalar field,

$$
\widetilde{Z_{s f s}^{\prime \prime 2}}=C_{v a r} \Delta^{2}|\nabla \widetilde{Z}|^{2} .
$$

The $\Delta$ parameter in Eq. (5) is the LES filter width. This model introduces a unique coefficient that can also be dynamically calculated using a variety of methods $[3,11]$. In all calculations shown here, $C_{v a r}$ is determined using the dynamic 'LED' procedure of Balarac et al. [11] and Lagrangian averaging [22].

\subsection{Transport Equation Models}

In a fully resolved setting, exact transport equations can be written for both the dissipation rate and the scalar variance. In the context of LES, however, several of the terms that appear in these equations require closure models. Here, the exact dissipation rate and variance equations are introduced first. The effect that a filtering operator has on these equations is then considered.

In reacting flows, the transport equation for $\chi_{Z}$ includes terms that depend on gradients of diffusivity and density. When this equation is solved in LES, these terms all require closure. To limit the appearance of unclosed terms, a transport equation for $|\nabla Z|^{2}$, rather than for $\chi_{Z}$, is considered. Letting $D / D t$ denote the material derivative, this equation is

$$
\begin{aligned}
\frac{D}{D t}\left(\rho|\nabla Z|^{2}\right)= & \frac{\partial}{\partial x_{j}}\left(\rho \mathcal{D}_{Z} \frac{\partial}{\partial x_{j}}\left(|\nabla Z|^{2}\right)\right) \\
& -2 \rho\left(\frac{\partial u_{i}}{\partial x_{j}} \frac{\partial Z}{\partial x_{i}} \frac{\partial Z}{\partial x_{j}}\right) \\
& -2 \rho \mathcal{D}_{Z}\left(\frac{\partial^{2} Z}{\partial x_{i} \partial x_{j}}\right)^{2} \\
& -\frac{2}{\rho} \frac{\partial \rho}{\partial x_{i}} \frac{\partial Z}{\partial x_{i}}\left(\frac{\partial}{\partial x_{j}}\left(\rho \mathcal{D}_{Z} \frac{\partial Z}{\partial x_{j}}\right)\right) \\
& +2 \frac{\partial\left(\rho \mathcal{D}_{Z}\right)}{\partial x_{i}} \frac{\partial Z}{\partial x_{i}}\left(\frac{\partial^{2} Z}{\partial x_{j} \partial x_{j}}\right) \\
& +2 \frac{\partial Z}{\partial x_{i}} \frac{\partial Z}{\partial x_{j}}\left(\frac{\partial^{2}\left(\rho \mathcal{D}_{Z}\right)}{\partial x_{i} \partial x_{j}}\right) .
\end{aligned}
$$


The $(a)$ and $(b)$ terms in Eq. (6) describe the production and dissipation, respectively, of the scalar dissipation rate. The $(c)$ through $(e)$ terms describe the effects of changes in density and diffusivity, and reduce to zero when $\rho$ and $\mathcal{D}_{Z}$ are constant. Once solved, the $|\nabla Z|^{2}$ quantity from Eq. (6) can be multiplied with $\mathcal{D}_{Z}$ to determine $\chi_{Z}$.

Applying an LES density weighted filter to Eq. (6) results in a transport equation for the term $\widetilde{\left.\nabla Z\right|^{2}}$. It will be assumed that correlations between mixture fraction gradients and the scalar diffusivity are small, so that this term can be similarly multiplied with the filtered diffusivity to compute the dissipation rate,

$$
2 \overline{\mathcal{D}_{Z}|\nabla Z|^{2}} \approx 2 \overline{\mathcal{D}}_{Z} \widetilde{|\nabla Z|^{2}} \text {. }
$$

Analysis from other researchers suggests that the density weighting which differentiates the filtering of $\widetilde{|\nabla Z|^{2}}$ and $\overline{|\nabla Z|^{2}}$ can be neglected on the grounds that it has a relatively small influence [23]. Calculations of both $\widetilde{|\nabla Z|^{2}}$ and $\overline{|\nabla Z|^{2}}$ in the DNS that will be introduced in section 3 confirmed this finding: dissipation rates computed using these differently weighted scalar gradients could not be statistically distinguished. Therefore, the density weighted term $\widehat{|\nabla Z|^{2}}$ will be used to calculate dissipation rates in the remainder of the study.

The equation for the filtered term $\widetilde{|\nabla Z|^{2}}$ may now be written,

$$
\begin{aligned}
& \frac{D}{D t}\left(\bar{\rho} \mid \widetilde{\left.\nabla Z\right|^{2}}\right)=\frac{\partial}{\partial x_{j}}\left(\bar{\rho}\left(\widetilde{\mathcal{D}}_{Z}+D_{t}\right) \frac{\partial}{\partial x_{j}}\left(\widetilde{|\nabla Z|^{2}}\right)\right) \\
& -2 \overline{\left(\frac{\partial u_{i}}{\partial x_{j}} \frac{\partial Z}{\partial x_{i}} \frac{\partial Z}{\partial x_{j}}\right)} \\
& -2 \rho \mathcal{D}_{Z}\left(\frac{\partial^{2} Z}{\partial x_{i} \partial x_{j}}\right)^{2} \\
& -\overline{\frac{2}{\rho} \frac{\partial \rho}{\partial x_{i}} \frac{\partial Z}{\partial x_{i}}\left(\frac{\partial}{\partial x_{j}}\left(\rho \mathcal{D}_{Z} \frac{\partial Z}{\partial x_{j}}\right)\right)} \\
& +2 \overline{\frac{\partial\left(\rho \mathcal{D}_{Z}\right)}{\partial x_{i}} \frac{\partial Z}{\partial x_{i}}\left(\frac{\partial^{2} Z}{\partial x_{j} \partial x_{j}}\right)} \\
& +2 \overline{\frac{\partial Z}{\partial x_{i}} \frac{\partial Z}{\partial x_{j}}\left(\frac{\partial^{2}\left(\rho \mathcal{D}_{Z}\right)}{\partial x_{i} \partial x_{j}}\right)},
\end{aligned}
$$

where the standard turbulent diffusivity model has been invoked to describe subfilter scalar flux,

$$
\bar{\rho} D_{t} \frac{\partial}{\partial x_{j}}\left(\widetilde{|\nabla Z|^{2}}\right)=\bar{\rho} \widetilde{u}_{j} \widetilde{|\nabla Z|^{2}}-\bar{\rho} u_{j} \widetilde{|\nabla Z|^{2}} .
$$

The (a) through (e) terms in Eq. (8) are unclosed. Sanders et al. [19] provides a thorough review of the relevant closure modeling for Reynolds averaged approaches. In LES, it can be argued that there is a need for somewhat different closures. This issue will be further addressed in section 5 .

The subfilter scalar variance can be solved for in a number of different ways. Based on Kaul et al.'s observations [12], a transport equation for $Z^{2}$ will be used here. In an unfiltered sense, $Z^{2}$ obeys the equation

$$
\frac{D}{D t}\left(\rho Z^{2}\right)=\frac{\partial}{\partial x_{j}}\left(\rho \mathcal{D}_{Z} \frac{\partial}{\partial x_{j}}\left(Z^{2}\right)\right)-\rho \chi_{Z} .
$$


After LES filtering, the equation becomes

$$
\frac{D}{D t}\left(\bar{\rho} \widetilde{Z^{2}}\right)=\frac{\partial}{\partial x_{j}}\left(\bar{\rho}\left(\widetilde{\mathcal{D}}_{Z}+\mathcal{D}_{t}\right) \frac{\partial}{\partial x_{j}}\left(\widetilde{Z^{2}}\right)\right)-\bar{\rho} \widetilde{\chi}_{Z}
$$

Equation (11) is noteworthy because it does not add to the closure modeling problem. Rather, if the scalar dissipation rate $\tilde{\chi}_{Z}$ has been closed, then Eq. (11) is closed. Once solved, Eq. (11) can be used to calculate the subfilter variance $\widetilde{Z_{s f s}^{\prime \prime 2}}$ using Eq. (3). It should be noted that numerical errors in the $\widetilde{Z^{2}}$ equation can have a significant influence on these variance predictions, and that the consideration of these errors is a subject of current research efforts.

\section{Auto-Igniting Jet}

\subsection{DNS Description}

The primary DNS case that will be used to assess the dissipation and variance models is an auto-igniting slot jet. This ethylene fueled flame was originally the subject of a DNS study by Yoo et al. [24]. A schematic of the flame is shown in Fig. 1, where velocity contours are plotted on the left and temperature contours are plotted on the right. The central fuel jet has a bulk velocity of $220 \mathrm{~m} / \mathrm{s}$, a temperature of $550 \mathrm{~K}$, and a composition of $82 \% \mathrm{~N}_{2}$ and $18 \% \mathrm{C}_{2} \mathrm{H}_{4}$ by volume. This central jet stream is denoted by the mixture fraction composition $Z=1$. The co-flow that surrounds the central jet consists of $100 \%$ air at a temperature of $1550 \mathrm{~K}$. The air co-flow enters the domain at a bulk velocity of 20 $\mathrm{m} / \mathrm{s}$, and this air stream is denoted by a mixture fraction of $Z=0$. No nozzle separates the main jet and co-flow; rather, the velocity, temperature, and composition profiles are prescribed to smoothly transition between $Z=0$ and $Z=1$ according to the functions listed in reference [24].
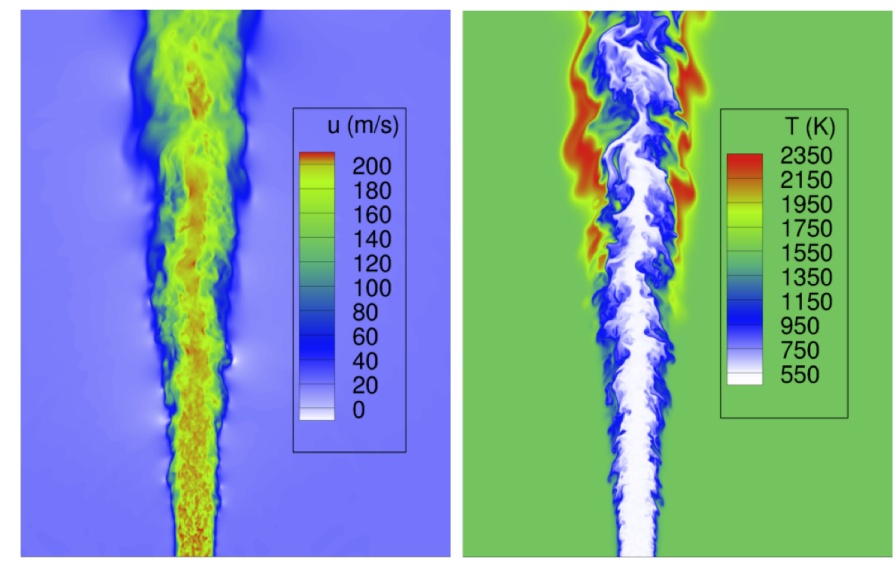

Figure 1: The ethylene flame DNS. Left: axial velocity contours; Right: temperature contours.

The total width of the central jet is $H=0.002 \mathrm{~m}$, and the domain size is $15 H \times 20 H \times 3 H$ in the downstream, cross-stream, and periodic directions, respectively. This same domain size will be used in all of the LES simulations shown below. The jet Reynolds number associated with the DNS conditions is 10,000, and the DNS is run at atmospheric pressure. The stoichiometric mixture fraction that results from the use of the diluted ethylene fuel 
stream is $Z_{s t}=0.27$. The DNS mesh consists of 1.3 billion cells, and the simulation required approximately 14 million CPU hours of run time.

Chemistry in the flame is described using a 22 species ethlyene mechanism [24] that was reduced from a larger hydrocarbon mechanism. This same 22 species mechanism will be used in all of the modeled LES calculations that are shown.

Turbulence is introduced into the jet DNS by extracting velocity information from a single realization of a homogeneous isotropic turbulence (HIT) field, and then superimposing this information on a bulk velocity profile at the inlet of the jet simulation. The turbulence field was synthesized by specifying length scales, magnitudes of velocity fluctuations, and the spectral energy density [25]. As time advances in the reacting jet DNS, the plane from which turbulence data is taken in the HIT field also advances. In the calculations shown below, the HIT data is filtered for LES using a filter width $\Delta$. The $\Delta$ parameter is set as the characteristic length scale that describes the LES mesh cells spanning the jet inlet. The filter width therefore changes between the coarse and fine LES simulations in conjunction with the mesh resolution. The LES-filtered HIT data is injected into the LES simulations as a boundary condition.

\subsection{LES Combustion Model}

Although combustion modeling is not the focus of this work, the combustion model that is employed in the LES will be briefly reviewed. In all LES simulations of the jet DNS, combustion is described using an unsteady flamelet approach similar to that of Ihme and See [20] and Pitsch [26]. The model is implemented by first solving the unsteady flamelet equations $[1,27]$ for the species $\phi_{k}$,

$$
\rho \frac{\partial\left(\phi_{k}\right)}{\partial \tau}=\frac{1}{\mathrm{Le}_{k}} \frac{\rho \chi_{Z}}{2} \frac{\partial^{2} \phi_{k}}{\partial Z^{2}}+\rho \dot{\omega}_{k}+\frac{1}{4}\left(\frac{1}{\mathrm{Le}_{k}}-1\right) \frac{\partial \phi_{k}}{\partial Z}\left[\frac{\partial\left(\rho \chi_{Z}\right)}{\partial Z}+\frac{\chi_{Z}}{\mathcal{D}_{Z}} \frac{\partial\left(\rho \mathcal{D}_{Z}\right)}{\partial Z}\right] .
$$

In Eq. (12) $Z$ is the mixture fraction variable and $\mathrm{Le}_{k}$ is the Lewis number of the $k^{t h}$ species. The initial conditions of the species $\phi_{k}$ in Eq. (12) are linear profiles representing non-reactive mixing between $Z=0$ and $Z=1$. The dissipation rate appearing in the equation is modeled as

$$
\chi_{Z}=\chi_{Z, \mathrm{ref}} \cdot \frac{f(Z)}{f\left(Z_{\mathrm{ref}}\right)},
$$

where $f$ is a prescribed function of $Z$ [1]. Unsteady flamelet solutions are generated for a variety of reference scalar dissipation rates. They are then tabulated as a function of $Z, \chi_{Z, r e f}$, and the progress variable $C$, which is defined as the sum of four species mass fractions,

$$
\mathrm{C}=\mathrm{Y}_{\mathrm{H}_{2} \mathrm{O}}+Y_{\mathrm{H}_{2}}+Y_{\mathrm{CO}}+Y_{\mathrm{CO}_{2}} .
$$

This progress variable behaves as a time-like coordinate in flamelet space. A series of reacting large eddy simulations have revealed that differential diffusion effects are important in the jet DNS, and non-unity Lewis numbers are therefore used for all species in the flamelet calculations. The Lewis numbers of the filtered mixture fraction and progress variable scalars that are transported in the LES calculations are set to unity.

Once generated, the unsteady solutions are convoluted with presumed PDFs for application in the LES. A beta PDF is presumed to describe $Z$, while a delta PDF is presumed to describe $\chi_{Z \text {,ref }}$. Additionally, it is assumed that a single unsteady flamelet solution is representative of the conditions in an LES mesh cell. Consequently, only the mean value of the progress variable is needed for modeling [3]. These assumptions lead to a tabulated LES chemistry database of the form

$$
\widetilde{\phi}_{k}=\widetilde{\phi}_{k}\left(\widetilde{Z}, \widetilde{Z^{\prime \prime 2}}, \chi_{Z, \mathrm{ref}}, \widetilde{C}\right)
$$


where $\phi_{k}$ is any reacting quantity of interest. The reference dissipation rate can be determined from the local unconditional LES dissipation rate $\bar{\chi}_{Z}$ by filtering Eq. (1),

$$
\chi_{Z, \mathrm{ref}}=\bar{\chi}_{Z} \cdot \frac{f\left(Z_{\mathrm{ref}}\right)}{\overline{f(Z)}}
$$

where $\overline{f(Z)}$ is determined by convoluting the function $f(Z)$ with a beta PDF.

Filtered $C$ and $Z$ scalars are transported in the LES, and the scalar dissipation rate and subfilter variance are calculated using models from section 2 . The tabulated chemistry database from Eq. (15) is accessed during the LES to determine chemical information such as the density and the progress variable source term.

\subsection{LES Description}

Two LES runs are performed using different mesh resolutions. The first mesh consists of 1 million $(1 \mathrm{M})$ cells, and corresponds to an LES filter width to Kolmogorov scale ratio of approximately $\Delta / \eta=8$. The second LES mesh consists of 23 million (23M) cells, and the corresponding length scale ratio is $\Delta / \eta=4.5$. These ratios are approximate because mesh stretching is used in both the DNS and LES, and because the turbulence intensity varies along the cross-stream direction. In regions surrounding the jet inlet and shear layer, the mesh stretching is weak and adjacent cell widths differ by less than one percent.

The DNS data is filtered for comparison with LES using coarse and fine filter widths that correspond to the 1 million cell and 23 million cell LES meshes, respectively. The filter widths $(\Delta)$ are determined by calculating the average characteristic LES cell length scale in the region spanning \pm 2 jet widths from the centerline and +8 jet widths downstream from the inlet. The calculated $\Delta$ values are then set to weakly increase in the downstream and radial directions in accordance with the LES mesh stretching.

Vervisch et al. [28] suggest that LES variance models should be tested at filter widths that are significantly smaller than time averaged scalar structures. If this condition is met, it can be safely assumed that interactions between the LES filtering operator and the time averaging operator do not affect scalar variance budgets. Both the coarse and fine LES ratios that are tested in this study were found to satisfy this condition with respect to the width of the time averaged mixture fraction shear layer profile.

The LES simulations are performed using a parallel finite-difference code [29]. The code is advanced in time using a Crank-Nicolson-type implicit scheme that is temporally second order accurate. Spatial gradients in the code are calculated using conservative schemes that are second order accurate for velocities and third order accurate for scalars. A conservative formulation is expected to be important for subfilter variance models based on $\widetilde{Z}$ and $\widetilde{Z^{2}}$ transport equations, because small differences in scalar magnitudes will affect scalar variance predictions to leading order. The code is run as a low Mach flow solver, so that a Poisson equation can be solved for the pressure variable to enforce conservation of mass.

Time averaged mixture fraction and velocity fields from the DNS and both LES cases are plotted in Fig. 2. The LES generally agrees well with the DNS, although two notable errors can be observed. The first is that the LES slightly under-predicts the width of the jet at the $\mathrm{X} / \mathrm{H}=3$ station. This error is seen to decrease with increasing LES mesh resolution, suggesting that it is attributable to the subfilter velocity model. The second error occurs at the $\mathrm{X} / \mathrm{H}=12$ station where the LES over-predicts the width of the jet. This second error is less sensitive to LES resolution and is the product of a slight vortex shedding instability that can be observed at downstream locations in the contour plots shown below in Fig. 3 . The over-prediction of the downstream jet width is partly a function of the LES model's treatment of cross stream boundary conditions, and of the jet entrainment that results from this boundary treatment. The shedding instability that causes the error may be inhibited in 
the DNS by compressibility effects [30] which are not captured by the LES low Mach solver. While noteworthy, these errors have only a marginal impact on the LES model's treatment of subfilter variance and dissipation.
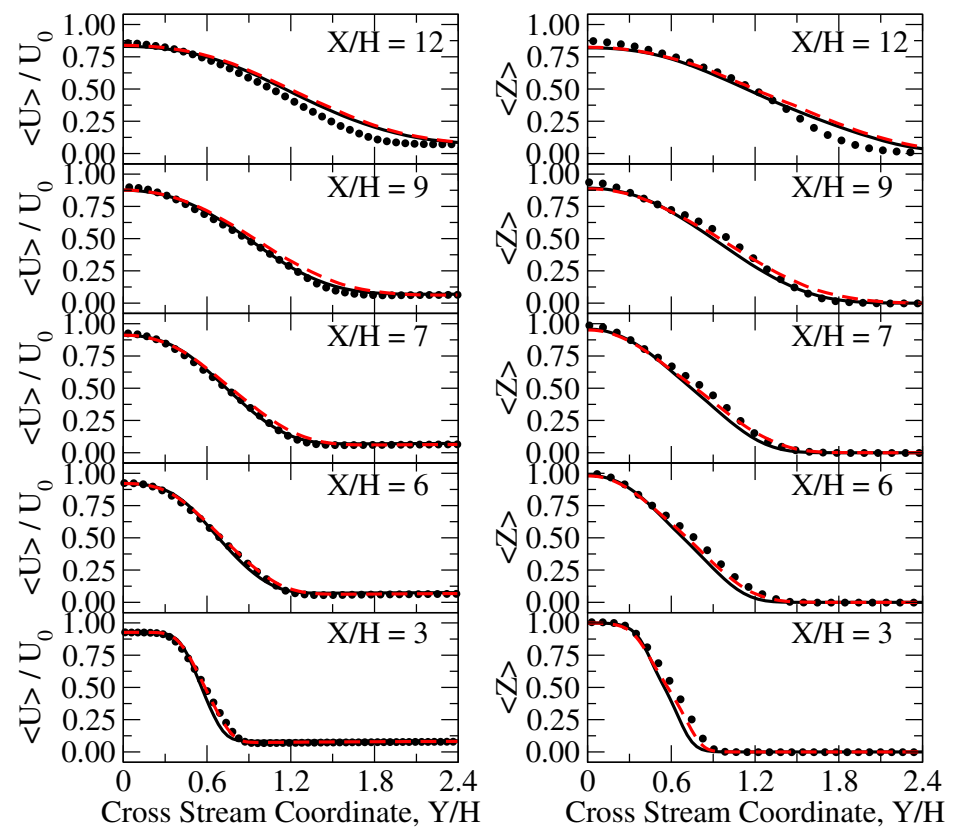

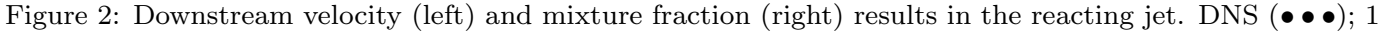
million cell LES $(-) ; 23$ million cell LES $(---)$.

\section{Algebraic Model Performance}

Figure 3 presents fields from the 1 million cell and 23 million cell LES runs alongside DNS fields that are filtered using the $\Delta$ from the 1 million cell LES. The filtered mixture fraction is plotted along with the algebraically modeled dissipation rate from Eq. (4) and the algebraically modeled subfilter scalar variance from Eq. (5). The $\widetilde{Z}$ field in the higher resolution LES is seen to capture significantly more flow structure than the lower resolution LES. Consequently, the burden on the subfilter models is eased in the higher resolution data set. The 1 million cell LES is therefore viewed as the primary subfilter modeling challenge.

Figure 4 compares the algebraically modeled scalar dissipation rate from Eq. (4) with the filtered dissipation rate from the DNS. All quantities are time averaged, as represented by the $\langle\cdot\rangle$ operator. Figure $4(\mathrm{a})$ shows $\bar{\chi}_{Z}$ as a function of the cross-stream coordinate at various downstream planes, while Fig. 4 (b) shows the mixture fraction conditioned $\bar{\chi}_{Z}$ in those same planes. The algebraic model's representation of $\bar{\chi}_{Z}$ is shown to be quite poor. At both LES resolutions, the model regularly under-predicts $\bar{\chi}_{Z}$ from the DNS. In the lower resolution case in particular, the under-predictions are order-of-magnitude differences. Because ignition is sensitive to local dissipation, these errors lead to significant under-predictions of the flame lift-off height. Although the $\bar{\chi}_{Z}$ predictions are improved when the LES resolution is increased, differences between the high and low resolution LES indicate that the algebraic model is undesirably sensitive to the filter size.

Resolved and subfilter contributions to the LES algebraic $\bar{\chi}_{Z}$ model are separated and compared with DNS data in Fig. 5. Both LES filter widths are considered. The resolved 


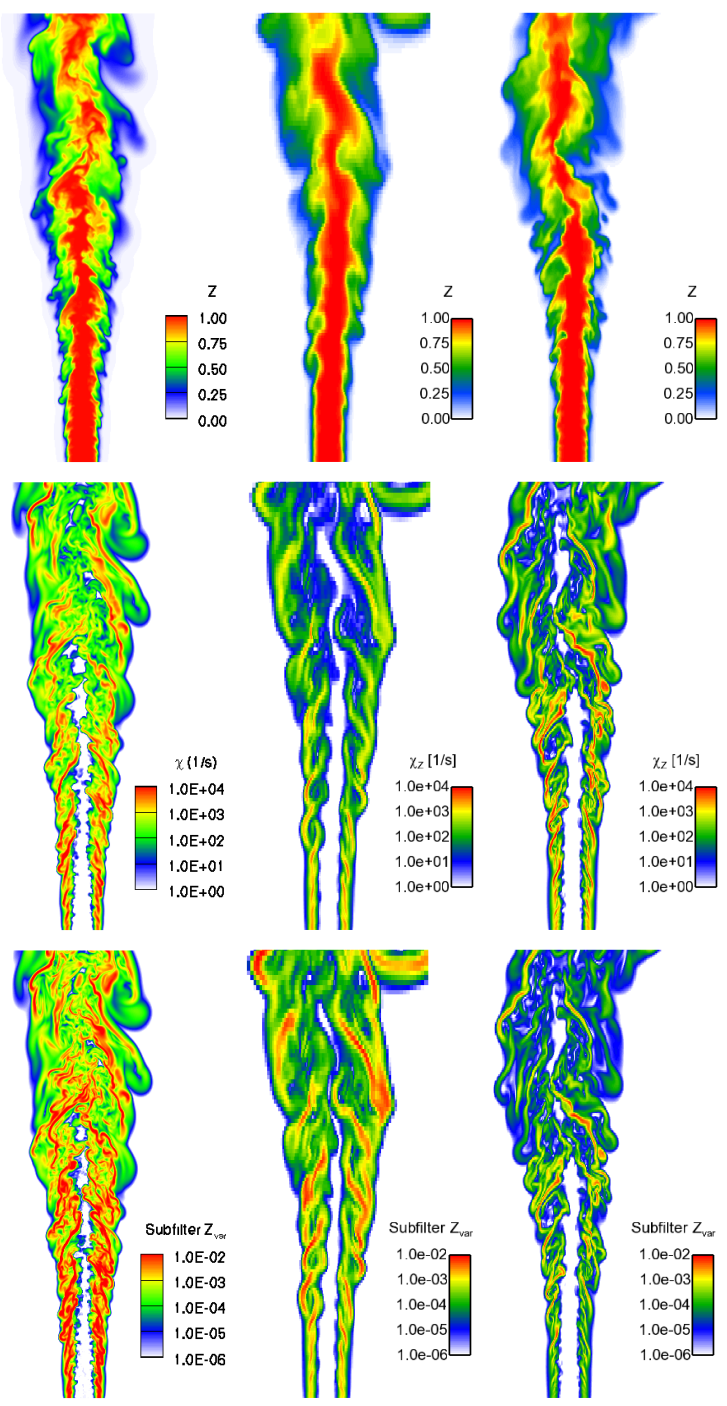

Figure 3: LES algebraic model results. Left column: DNS fields filtered using the 1M cell LES filter width; Middle column: $1 \mathrm{M}$ cell LES; Right column: $23 \mathrm{M}$ cell LES. Upper row: $\widetilde{Z}$ contours; Middle row: $\bar{\chi}_{Z}$ from Eq. (4); Lower row: $\widehat{Z_{s f s}^{\prime \prime 2}}$ from Eq. (5).

component of dissipation is calculated as $\left\langle 2 \overline{\mathcal{D}}_{Z}|\nabla \widetilde{Z}|^{2}\right\rangle$ while the subfilter component is calculated as $\left\langle\overline{2 \mathcal{D}_{Z}|\nabla Z|^{2}}-2 \overline{\mathcal{D}}_{Z}|\nabla \widetilde{Z}|^{2}\right\rangle$ and $\left\langle 2 \mathcal{D}_{t}|\nabla \widetilde{Z}|^{2}\right\rangle$ in the DNS and LES, respectively.

Figure 5(a) plots the dissipation rate components that are associated with the coarse filter width. At this filter resolution, the resolved dissipation from the filtered DNS (solid black circles) and subfilter dissipation from the filtered DNS (open blue circles) are comparable in magnitude. Subfilter dissipation modeling therefore plays an important role in the 1 million cell LES, even though the Reynolds number of the case is not representative of realistic combustor conditions. The resolved DNS dissipation rate is reasonably well described by the resolved component of the LES model (black solid line), at least in the rich part of the flame where turbulence is most intense. The subfilter LES dissipation (blue dashed line), however, universally underestimates the subfilter dissipation seen in the DNS. This subfilter 


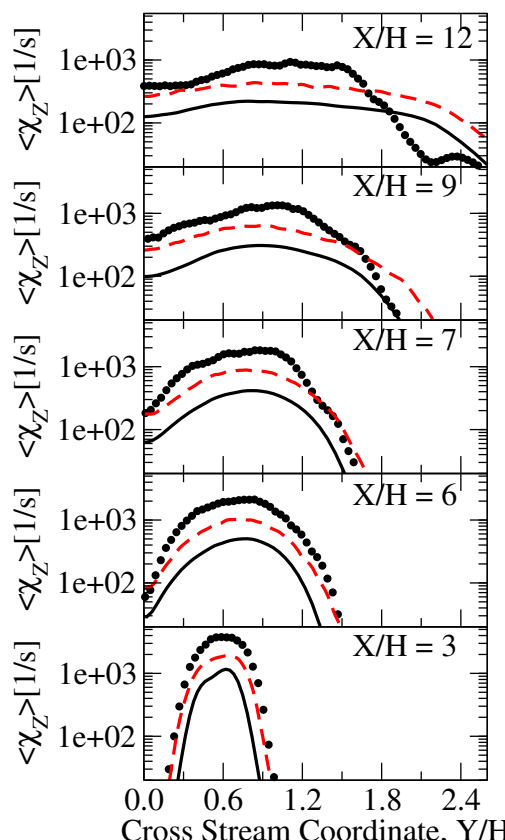

(a) physical space

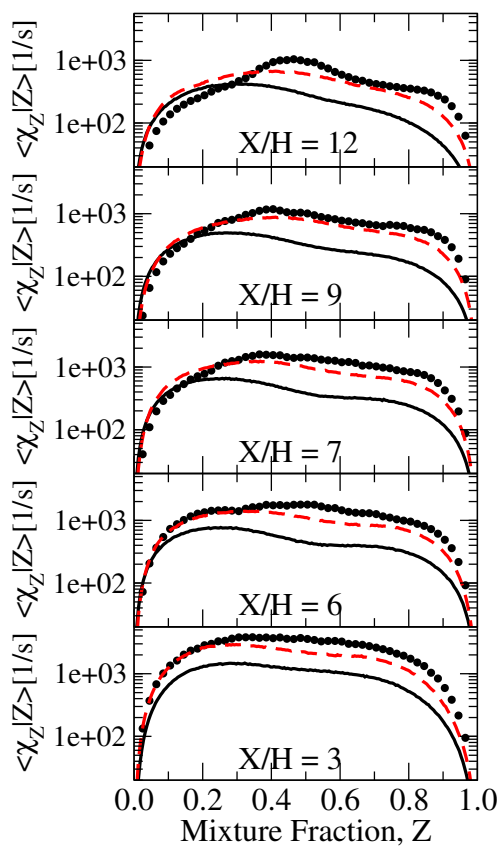

(b) mixture fraction space

Figure 4: Algebraic $\bar{\chi}_{Z}$ model results in the reacting jet, plotted in both (a) physical and (b) conditional

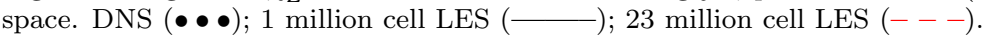

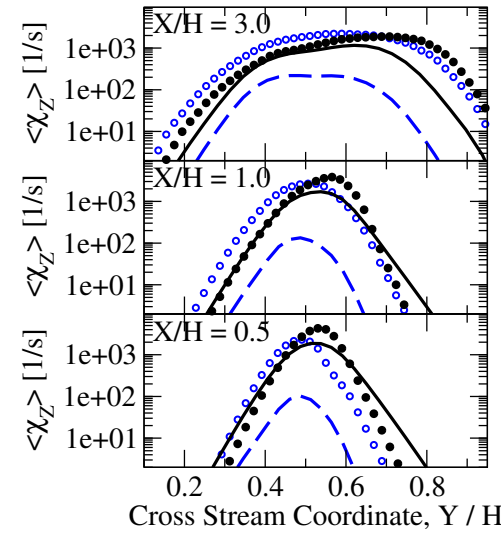

(a) 1 million cell LES filter width

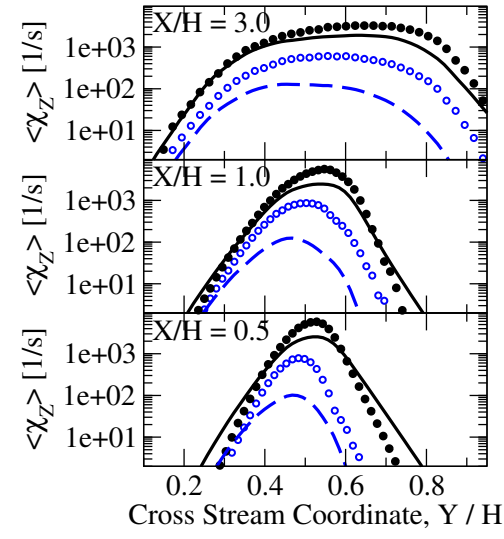

(b) 23 million cell LES filter width

Figure 5: Budget of the algebraic $\bar{\chi}_{Z}$ model in the reacting jet. Resolved dissipation from the filtered

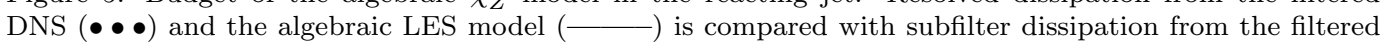
DNS ( $\circ \circ \circ)$ and the algebraic LES model $(---)$ at the: (a) 1 million cell filter width; (b): 23 million cell filter width.

modeling error is responsible for the bulk of the error in the total modeled dissipation rate.

Figure 5(b) demonstrates that the subfilter component of the algebraic model performs poorly even when the filter width is decreased. In spite of this poor performance, the total dissipation rate is predicted more accurately (see Fig. 4). The explanation for the 
improvement is that the dissipation rate budget shifts from the subfilter scales to the resolved scales as the filter width is decreased. The importance of the subfilter model therefore reduces in importance. Dissipation rate predictions in the 23 million cell LES can therefore be said to improve relative to the 1 million cell LES predictions in spite of, and not because of, subfilter model performance.

Three distinct sources of error can influence the subfilter dissipation rate that is predicted by the algebraic model. A first source of error is that the equilibrium assumption used to derive the algebraic model [13] may be locally violated. A second source of error is that even a perfectly predicted turbulent diffusivity may not describe the variance production term appearing in the equilibrium assumption. A third source of error is that the turbulent diffusivity in the production term may not be correctly modeled by a dynamic Smagorinsky approach. If either of the first two sources of error dominate the behavior of the algebraic model, then higher accuracy can be obtained only if the model form shown in Eq. (4) is changed. If the third source of error is dominant, however, then accuracy might be improved by using a different description of the turbulent diffusivity $\mathcal{D}_{t}$.

Time averaged diffusivities from the LES and DNS are plotted in Fig. 6 to determine the importance of these different sources of error. The turbulent diffusivity $\mathcal{D}_{t}$ that is calculated from the exact subfilter mixture fraction flux in the DNS is plotted using solid circles, while the modeled turbulent diffusivity from LES is plotted as a dashed line. The coarse and fine filter widths are considered in Figs. 6(a) and 6(b), respectively. For comparison, the molecular diffusivity $\mathcal{D}_{Z}$ from the LES is plotted as a solid line.

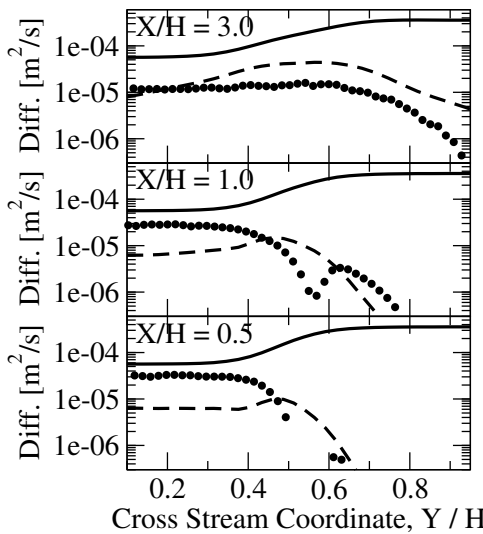

(a) 1 million cell LES filtter width

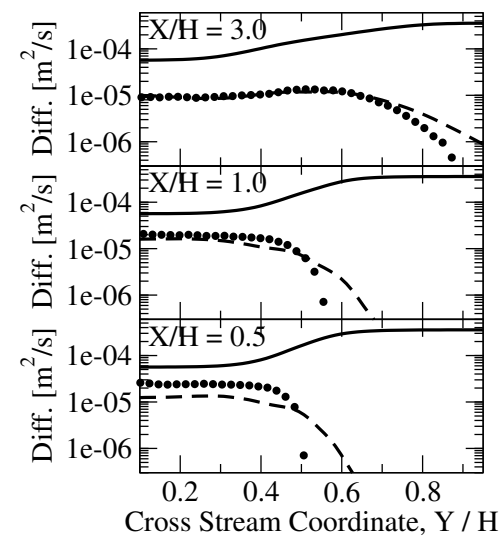

(b) 23 million cell LES filtter width

Figure 6: Time averaged diffusivities in the reacting jet. The turbulent diffusivity $\mathcal{D}_{t}$ from the filtered DNS

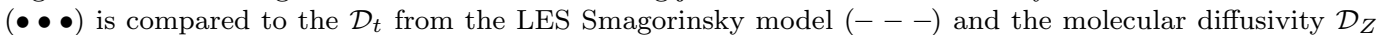
( $($ ). (a): 1 million cell filter width; (b): 23 million cell filter width.

The diffusivity data demonstrates that the algebraic model's under-prediction of subfilter dissipation cannot be attributed to an incorrectly predicted turbulent diffusivity. At values of the cross stream coordinate greater than $\mathrm{Y} / \mathrm{H}=0.5$, the LES turbulent diffusivity is greater than or equal to the diffusivity calculated from the DNS, regardless of the filter size. In these regions, a perfectly predicted turbulent diffusivity would lower the algebraic subfilter dissipation rate predictions in Fig. 5 even further away from the calculated DNS values. The only regions where the LES model under-predicts the turbulent diffusivity are located near the inlet at cross stream coordinates of less than $\mathrm{Y} / \mathrm{H}=0.4$. These regions correspond to the innermost portion of the jet shear layer, where the mixture fraction field is still fairly uniform and the dissipation rate is relatively small. Even in these regions, the turbulent 
diffusivity is under-predicted by a factor of no more than three. This difference cannot be used to explain the order of magnitude subfilter dissipation errors that are present in Fig. 5. Consequently, the accuracy of the subfilter dissipation model can be improved only if the form of this model is changed.

Figures 7 and 8 compare scalar variance predictions from the LES cases with the corresponding variance data from the DNS. Figure 7 (a) plots the time averaged resolved variance, $\left\langle\widetilde{Z}^{2}\right\rangle-\langle\widetilde{Z}\rangle^{2}$, associated with the coarse LES filter, while Fig. 7(b) plots the variance information that is resolved in when the finer LES filter is used. The subfilter variance $\left\langle\widetilde{Z_{s f s}^{\prime \prime 2}}\right\rangle$ that is associated with these two filter widths is plotted in Figure 8.

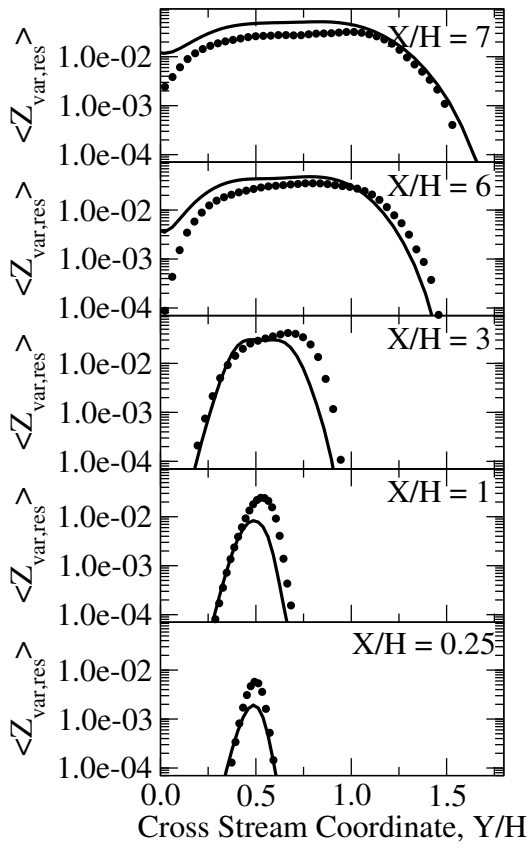

(a) 1 million cell LES filter width

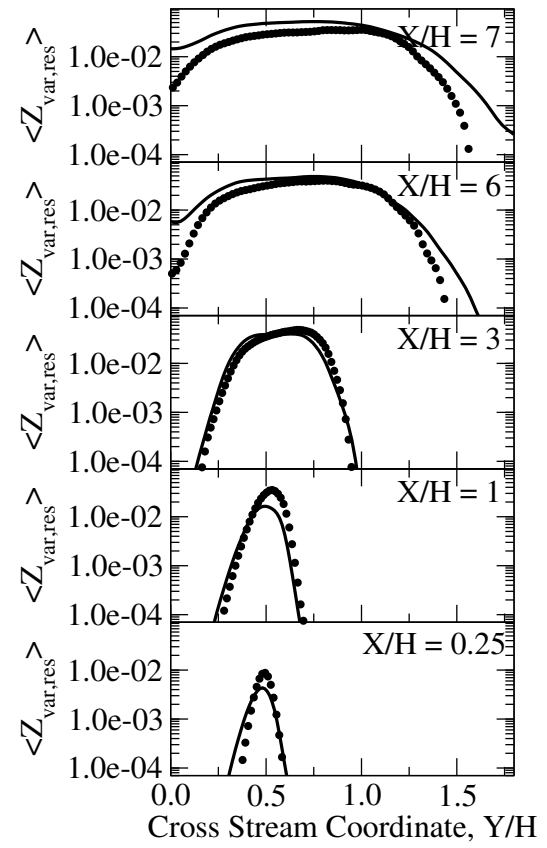

(b) 23 million cell LES filter wdith

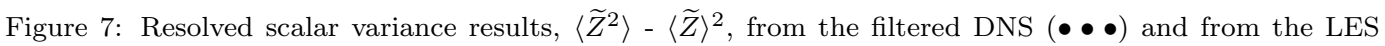
$(-)$ at the: (a) 1 million cell LES filter width; (b): 23 million cell LES filter width.

Scalar variance in the coarse mesh LES is similar to dissipation in that the resolved component of the variance is adequately captured, while the subfilter variance is not. Specifically, the subfilter algebraic variance model under-predicts the DNS data by as much as an order of magnitude. Under-predictions are more pronounced near the jet inlet, where the mixture fraction shear layer is transitioning from a laminar inlet profile to a fully turbulent mixing layer. The algebraic variance model performs more accurately at the finer LES resolution, and Fig. 8(a) indicates that even the subfilter variance is well predicted beyond the $\mathrm{X} / \mathrm{H}=6$ station. Under-predictions on the order of $100 \%$ are still observed near the jet inlet, however.

\section{An Adapted Dynamic LES Closure For The Dissipation Equation}

The algebraic dissipation rate and variance models that have been presented have been shown to be subject to several shortcomings. These shortcomings should not to be interpreted to mean that algebraic models are incapable of describing subfilter quantities. 


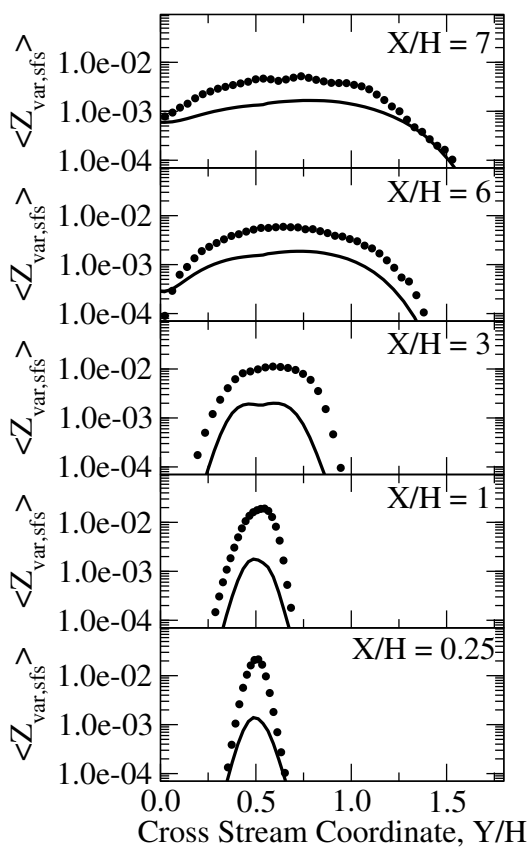

(a) 1 million cell mesh

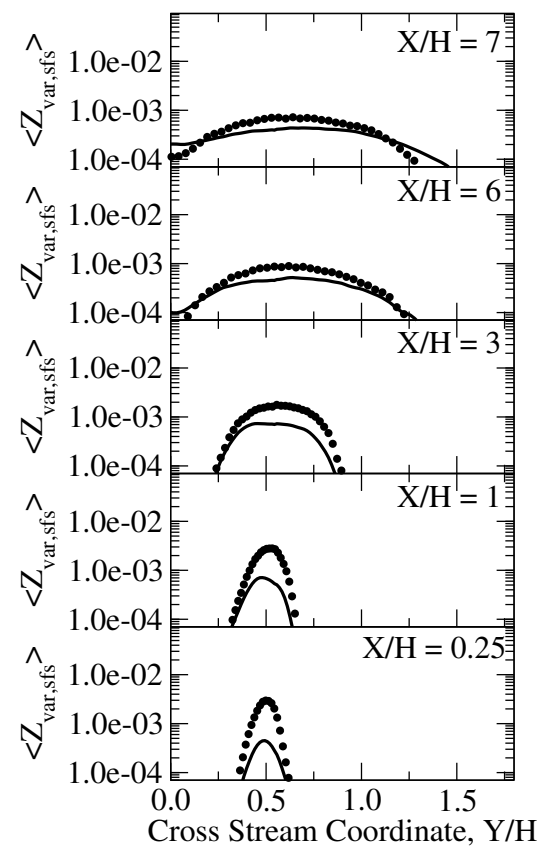

(b) 23 million cell mesh

Figure 8: Subfilter variance results, $\left\langle\widetilde{Z_{s f s}^{\prime \prime 2}}\right\rangle$, from the filtered DNS $(\bullet \bullet \bullet)$ and LES algebraic model $(-)$ at the: (a) 1 million cell LES filter width; (b): 23 million cell LES filter width.

Rather, the observed shortcomings are specific to the particular algebraic model realization that is considered here. One means of addressing these errors would be to adjust the form of the algebraic model equation $[10,14]$. Here, however, the transport equations that were shown in section 2 are considered as a means of improving dissipation and variance predictions. The use of transport equations allows the importance of production and dissipation imbalances to be explicitly accounted for. This section addresses the closure of the LES transport equation for $\widetilde{\left.\nabla Z\right|^{2}}$, shown in Eq. (8). Once closed, this equation can be used to calculate the dissipation rate $\bar{\chi}_{Z}$, which in turn can be used to close the transport equation for the $\widetilde{Z^{2}}$ variable that appears in Eq. (11).

\subsection{Production And Dissipation Closure Assumptions}

Five unclosed terms labeled $(a)$ through $(e)$ appear in the dissipation rate equation, Eq. (8). The two most important of these are the production and dissipation terms. They are split into resolved and subfilter components for use in LES,

$$
\begin{aligned}
& -2 \overline{\overline{(}\left(\frac{\partial u_{i}}{\partial x_{j}} \frac{\partial Z}{\partial x_{i}} \frac{\partial Z}{\partial x_{j}}\right)}=-2 \bar{\rho}\left(\frac{\partial \widetilde{u}_{i}}{\partial x_{j}} \frac{\partial \widetilde{Z}}{\partial x_{i}} \frac{\partial \widetilde{Z}}{\partial x_{j}}\right)+\mathcal{P}_{s f s} \\
& -2 \bar{\rho} \widetilde{\mathcal{D}}_{Z} \overline{\left(\frac{\partial^{2} Z}{\partial x_{i} \partial x_{j}}\right)^{2}}=-2 \bar{\rho} \widetilde{\mathcal{D}}_{Z}\left(\frac{\partial^{2} \widetilde{Z}}{\partial x_{i} \partial x_{j}}\right)^{2}-\Omega_{s f s}
\end{aligned}
$$


Following Reynolds averaged modeling approaches such as those reviewed by Sanders [19, 31], the subfilter production and dissipation terms will be written for LES as

$$
\begin{aligned}
& \mathcal{P}_{s f s}=C_{p r d} \cdot \bar{\rho} \cdot\left(\frac{u_{\Delta}^{\prime 3}}{\Delta \cdot \nu_{t}}\right)^{1 / 2} \cdot\left(\widetilde{|\nabla Z|^{2}}-|\nabla \widetilde{Z}|^{2}\right), \\
& \Omega_{s f s}=C_{d i s} \cdot \bar{\rho} \cdot \frac{\widetilde{\mathcal{D}}_{Z}}{\widetilde{Z_{s f s}^{\prime \prime 2}}} \cdot\left(\widetilde{|\nabla Z|^{2}}-|\nabla \widetilde{Z}|^{2}\right)^{2} .
\end{aligned}
$$

$C_{p r d}$ and $C_{d i s}$ are model coefficients, and $\widetilde{Z_{s f s}^{\prime \prime 2}}$ is the subfilter scalar variance that is modeled either algebraically or with a transport equation. $\nu_{t}$ is the turbulent viscosity that, in the following sections, will be calculated using a dynamic Smagorinsky model with Lagrangian averaging [22]. The $u_{\Delta}^{\prime 3} / \Delta$ term in the production model can be viewed as the subfilter momentum dissipation, $\epsilon_{\Delta}$. In the homogeneous DNS calculations presented next, $u_{\Delta}^{\prime 3}$ is calculated by estimating a velocity fluctuation from the calculated subfilter kinetic energy. In the LES calculations of the reacting jet that are shown later, $u_{\Delta}^{\prime 3} / \Delta$ is calculated following Deardorff [32] as

$$
\frac{u_{\Delta}^{\prime} 3}{\Delta} \approx \frac{\left(\nu_{t} /(0.1 \cdot \Delta)\right)^{3}}{\Delta}=1000 \cdot \frac{\nu_{t}^{3}}{\Delta^{4}} .
$$

Any similar model could be used to describe $u_{\Delta}^{\prime}$, if desired. The LES subfilter production term that results from the use of Deardorff's model is

$$
\mathcal{P}_{s f s}=C_{p r d} \cdot \bar{\rho} \cdot \frac{32 \cdot \nu_{t}}{\Delta^{2}} \cdot\left(\widetilde{|\nabla Z|^{2}}-|\nabla \widetilde{Z}|^{2}\right) .
$$

It will be assumed that the remaining $(c)$ through $(e)$ terms in Eq. (8) are of less importance on subfilter scales. This assumption can be argued for on the grounds that the mixture fraction $Z$, mixture fraction gradient $\partial Z / \partial x_{i}$, and molecular diffusivity $\mathcal{D}_{Z}$ are expected to be less correlated with one another than the quantities in terms $(a)$ and $(b)$ are. The subfilter modeling of terms $(c)$ through $(e)$ will therefore be neglected in the remainder of the study. This is an important assumption that should be revisited in future work. 


\subsection{Modeled LES Scalar Dissipation Equation}

These dissipation closure assumptions lead to the following form of the $\widetilde{|\nabla Z|^{2}}$ equation,

$$
\begin{aligned}
\frac{D}{D t}\left(\bar{\rho} \mid \widetilde{\left.\nabla Z\right|^{2}}\right) & =\frac{\partial}{\partial x_{j}}\left(\bar{\rho}\left(\widetilde{\mathcal{D}}_{Z}+D_{t}\right) \frac{\partial}{\partial x_{j}}\left(\widetilde{\left.\nabla Z\right|^{2}}\right)\right) \\
& -2 \bar{\rho}\left(\frac{\partial \widetilde{u}_{i}}{\partial x_{j}} \frac{\partial \widetilde{Z}}{\partial x_{i}} \frac{\partial \widetilde{Z}}{\partial x_{j}}\right) \\
& -2 \bar{\rho} \widetilde{\mathcal{D}}_{Z}\left(\frac{\partial^{2} \widetilde{Z}}{\partial x_{i} \partial x_{j}}\right)^{2} \\
& -\frac{2}{\bar{\rho}} \frac{\partial \bar{\rho}}{\partial x_{i}} \frac{\partial \widetilde{Z}}{\partial x_{i}}\left(\frac{\partial}{\partial x_{j}}\left(\bar{\rho} \widetilde{\mathcal{D}}_{Z} \frac{\partial}{\partial x_{j}}(\widetilde{Z})\right)\right) \\
& +2 \frac{\partial\left(\bar{\rho} \widetilde{\mathcal{D}}_{Z}\right)}{\partial x_{i}} \frac{\partial \widetilde{Z}}{\partial x_{i}}\left(\frac{\partial^{2} \widetilde{Z}}{\partial x_{j} \partial x_{j}}\right) \\
& +2 \frac{\partial \widetilde{Z}}{\partial x_{i}} \frac{\partial \widetilde{Z}}{\partial x_{j}}\left(\frac{\partial^{2}\left(\bar{\rho} \widetilde{\mathcal{D}}_{Z}\right)}{\partial x_{i} \partial x_{j}}\right) \\
& +C_{p r d} \cdot \bar{\rho} \cdot \frac{32 \cdot \nu_{t}}{\Delta^{2}} \cdot\left(\widetilde{|\nabla Z|^{2}}-|\nabla \widetilde{Z}|^{2}\right) \\
& -C_{d i s} \cdot \bar{\rho} \cdot \frac{\widetilde{\mathcal{D}}_{Z}}{\widetilde{Z_{s f s}^{\prime \prime 2}}} \cdot\left(\widetilde{|\nabla Z|^{2}}-|\nabla \widetilde{Z}|^{2}\right)^{2} .
\end{aligned}
$$

Once closed, $\widetilde{|\nabla Z|^{2}}$ from Eq. (23) is multiplied with $\widetilde{\mathcal{D}}_{Z}$ to determine $\bar{\chi}_{Z}$. This $\bar{\chi}_{Z}$ value can then be used as the source term in the subfilter variance equation, Eq. (11). The only remaining barrier to implementation of the transport equation models for the dissipation and variance is the specification of $C_{p r d}$ and $C_{d i s}$.

\subsection{Scalar Mixing Turbulence Database}

Closure models for $C_{p r d}$ and $C_{d i s}$ are especially difficult to develop because most of the dissipation rate's energetic content exists on the smallest scales of a flow. This small scale content inhibits the accuracy of traditional dynamic LES approaches for describing model coefficients, which tend to extrapolate information from large resolved scales to small unresolved scales. When the large scales contain negligible amounts of information relative to the small scales, the extrapolation procedure ceases to aid in modeling. Alternatives to the traditional dynamic algorithm are therefore needed.

The evaluation of $C_{p r d}$ and $C_{d i s}$ will be investigated using a second database of direct numerical simulations that describe conserved scalar mixing in the presence of forced, constant density, homogeneous isotropic turbulence. This second set of DNS data was originally developed in order to study probability distribution functions describing multi-scalar mixing [33]. These DNS cases are run on a $512^{3}$ computational mesh and are forced using the scheme of Rosales and Meneveau [34] to a Reynolds number of approximately $\operatorname{Re}_{\lambda}=100$. Scalar fields are then initialized in the domain according to the procedure of Eswaran et al. [7]. This initialization procedure first sets the scalar field to have a single, constant value over the entire DNS domain. A series of integral scale patches are then identified [7], and the scalar field within these patches is set to a second constant value. In scalar phase space, these initial fields are characterized by a double delta function, where the two delta function peaks are located at the background and patch scalar values, respectively. The DNS 

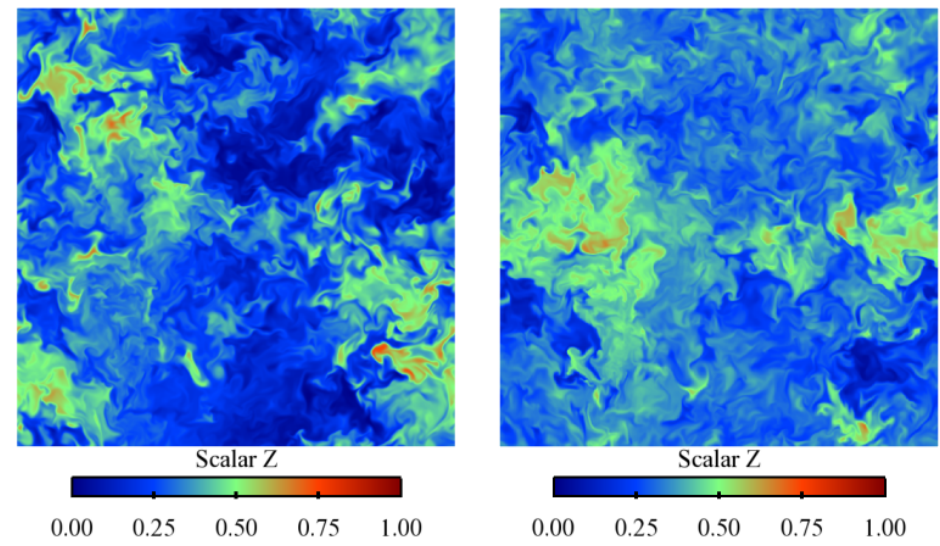

Figure 9: Typical planes from two of the the $512^{3}$ scalar mixing homogeneous turbulence DNS runs [33], showing contours of the conserved scalar.

simulation is then continued, and the scalar fields are allowed to mix towards homogeneity. Further details regarding the scalar mixing DNS can be found in references [33, 35].

Data is extracted from the homogeneous DNS for analysis at one eddy turnover time after the scalar field is initialized. Typical contour planes that show the scalar field at this point in time appear in Fig. 9. This scalar data is filtered for analysis using clipped Gaussian filter kernels [36]. The filtering is performed at a variety of filter widths, and model results are parameterized by these widths. The filtered data can be used to assess the accuracy of models for $C_{p r d}$ and $C_{d i s}$.

Unlike the reacting jet DNS that was introduced in section 3, the homogeneous DNS allows field data to be analyzed over a wide range of LES filter widths. The reacting jet imposes an upper limit on LES filter widths because any LES of a jet case must adequately resolve the momentum profile that form across the jet. Conversely, LES filter widths in the homogeneous DNS are only limited by the maximum size of the domain. A subfilter model should ideally be valid over a wide range of LES filter widths. Filter ratios up to $\Delta / \eta=128$ are therefore analyzed in the homogeneous case, even though this same range of filters cannot be reasonably tested in a posteriori LES of the reactive jet case.

\subsection{Model Coefficient PDFs}

Figure 10 shows the PDFs that describe the distribution of the model coefficient $C_{\text {prd }}$ after one eddy turnover time in the scalar mixing DNS. This coefficient is evaluated at each location in the homogeneous DNS domain using the formula

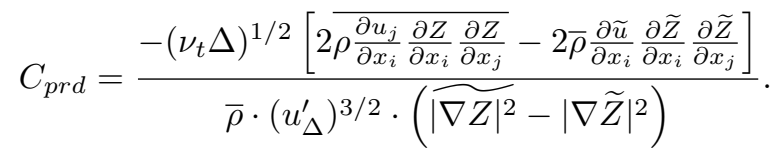

PDFs of the coefficient are constructed for a given filter size by binning results from the entire domain.

Figure 10 shows that the $C_{p r d}$ PDF is uni-modal. Additionally, the value of the model coefficient is reasonably filter independent: PDFs associated with different filter widths have approximately the same mean, and relax towards a single value as the filter width increases. $C_{p r d}$ is therefore reasonably represented by a single, filter-independent constant. In agreement with several Reynolds averaged modeling approaches [19], Fig. 10 indicates that this coefficient should have a value of unity. The value $C_{p r d}=1.0$ is therefore adopted. 


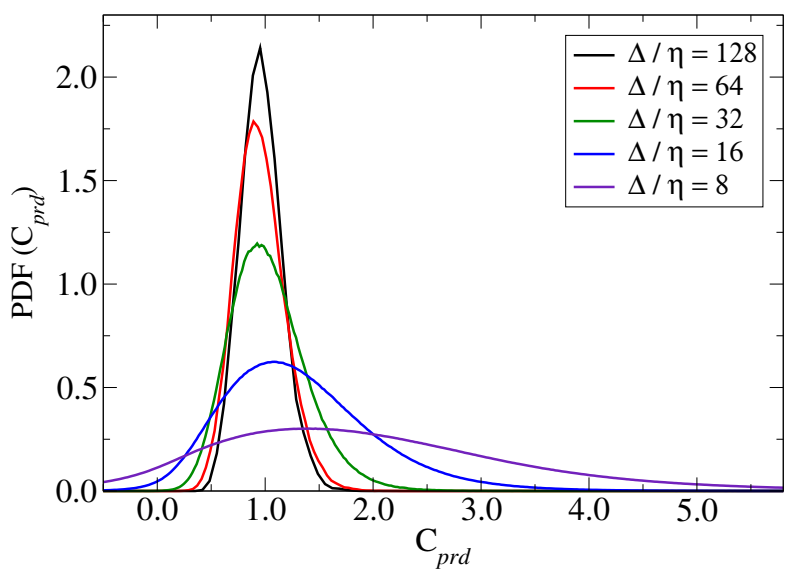

Figure 10: PDFs of the model coefficient $C_{\text {prd }}$ from Eq. (23), evaluated from the homogeneous scalar mixing DNS. The PDFs are parameterized by the LES filter width, $\Delta$.

PDFs of the model coefficient $C_{d i s}$ are shown in Fig. 11. $C_{d i s}$ is calculated at each location in the DNS domain as

$$
C_{d i s}=\frac{\left.\widetilde{Z_{s f s}^{\prime \prime 2}\left[2 \rho \mathcal{D}_{Z}\left(\frac{\partial^{2} Z}{\partial x_{i} \partial x_{j}}\right)^{2}\right.}-2 \bar{\rho} \widetilde{\mathcal{D}}_{Z}\left(\frac{\partial^{2} \widetilde{Z}}{\partial x_{i} \partial x_{j}}\right)^{2}\right]}{2 \widetilde{\mathcal{D}}_{Z}\left(\widetilde{|\nabla Z|^{2}}-|\nabla \widetilde{Z}|^{2}\right)^{2}} .
$$

The PDFs in Fig. 11 are again unimodal, but the value of $C_{d i s}$ is filter dependent. As the LES filter width increases, $C_{d i s}$ increases. Consequently, this coefficient cannot be described as a single value. Filter dependencies such as these are often dealt with by employing dynamic models. As discussed above, however, the dynamic procedure is not applicable to quantities like $|\nabla Z|^{2}$ whose energy content exists on small flow scales.

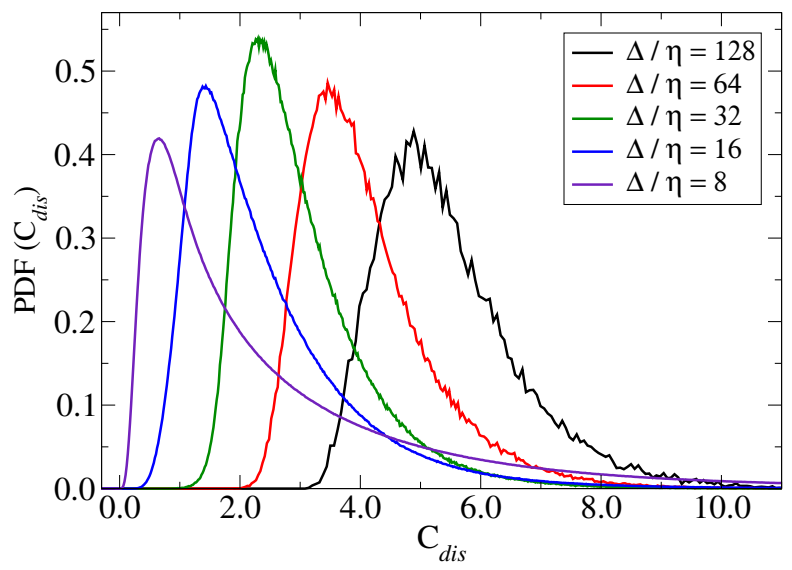

Figure 11: PDFs of $C_{\text {dis }}$ from Eq. (23), evaluated from the homogeneous scalar mixing DNS. The PDFs are parameterized by the LES filter width $\Delta$. 


\subsection{Adapted Dynamic LES Closure}

Here an alternative to the traditional dynamic approach is proposed. This alternative is rooted in the recognition that many turbulence modeling coefficients respond similarly to changes in the local intensity of turbulence. For example, if the passage of a turbulent eddy increases the local turbulent scalar flux, the scalar variance might be expected to increase in the same way. Similarly, a flow event that causes the local scalar variance to increase might also increase the local dissipation rate. Viewed from the reverse perspective, subfilter model coefficients should tend toward zero in flow regions that laminarize or that are adjacent to walls.

The idea of the adapted dynamic closure is to take information from quantities that are amenable to dynamic calculations, and to apply that information to other quantities that are not similarly amenable. This projection of information is useful because small scale quantities are, like large scale quantities, sensitive to local turbulence. Information regarding local turbulence can be used to increase the accuracy of a model coefficient regardless of where the modeled quantity's spectrum peaks.

This projection of information is possible because an appropriate value of the model coefficient must exist. Indeed, the problem with applying a standard dynamic procedure to small scale quantities is not that the coefficients are indeterminate. Rather, the problem is that coefficient values cannot be calculated due to the absence of useful large scale information. The adapted dynamic closure simply circumvents this calculation problem by borrowing information from other readily obtained dynamic solutions.

The adapted dynamic closure can be used in the scalar mixing DNS to compute the coefficient $C_{d i s}$. Dynamic information will be borrowed from the algebraic scalar variance model in Eq. (5), and $C_{d i s}$ will be rewritten as,

$$
C_{d i s}=C_{d i s, d} \cdot\left(\frac{C_{v a r}}{C_{v a r}^{0}}\right)
$$

$C_{d i s, d}$ in this expression is now the unknown model coefficient, while $C_{v a r}$ is the dynamically computed variance coefficient from Eq. (5). $C_{v a r}^{0}$ is a baseline value of the variance coefficient that can be determined by using a Taylor series expansion to derive the algebraic variance model [11]. Proceeding through this derivation leads to $C_{v a r}^{0}=1 / 12$. This number can be viewed as the variance coefficient's value in a particular turbulence regime. The details of this regime, and of its relationship to homogeneous turbulence, are irrelevant. These details will be accounted for in the specification of the constant $C_{d i s, d}$. The intensity of $C_{v a r}$ relative to this referenced regime is the only information of interest, and this information is captured by the ratio in Eq. (26).

The adapted dynamic closure is tested by using the scalar mixing DNS to calculate PDFs of the coefficient $C_{d i s, d}$. Coefficients are determined by first using the DNS to determine the exact value of $C_{v a r}$ at all locations in the DNS domain. $C_{d i s, d}$ is then determined from the expression

$$
C_{d i s, d}=\left(\frac{C_{v a r}^{0}}{C_{\text {var }}}\right) \frac{\widetilde{Z_{s f s}^{\prime \prime 2}}\left[2 \rho \overline{\mathcal{D}_{Z}\left(\frac{\partial^{2} Z}{\partial x_{i} \partial x_{j}}\right)^{2}}-2 \bar{\rho} \widetilde{\mathcal{D}}_{Z}\left(\frac{\partial^{2} \widetilde{Z}}{\partial x_{i} \partial x_{j}}\right)^{2}\right]}{2 \widetilde{\mathcal{D}}_{Z}\left(\widetilde{|\nabla Z|^{2}}-|\nabla \widetilde{Z}|^{2}\right)^{2}},
$$

where $C_{v a r}^{0}=1 / 12$. The subfilter variance $\widetilde{Z_{s f s}^{\prime \prime 2}}$ in this expression is computed directly from the DNS, and the coefficient $C_{v a r}$ is computed so that the algebraic subfilter variance model is exact. Equation (27) differs from the expression used to calculate $C_{d i s}$ only in that it is multiplied by the ratio $\left(C_{v a r} / C_{v a r}^{0}\right)$. 
PDFs describing the distribution of $C_{d i s, d}$ in the scalar mixing DNS are plotted in Fig. 12. These distributions are largely independent of the LES filter width, and are unimodal. The information that was provided by the dynamic variance calculation therefore correctly represented the filter dependent component of the $C_{d i s}$ coefficient. The removal of this information reduces the amount of physical information that a model coefficient must attempt to describe. When the adapted dynamic procedure is incorporated into the modeling framework, the remaining coefficient $C_{d i s, d}$ can be accurately treated as a single, constant value.

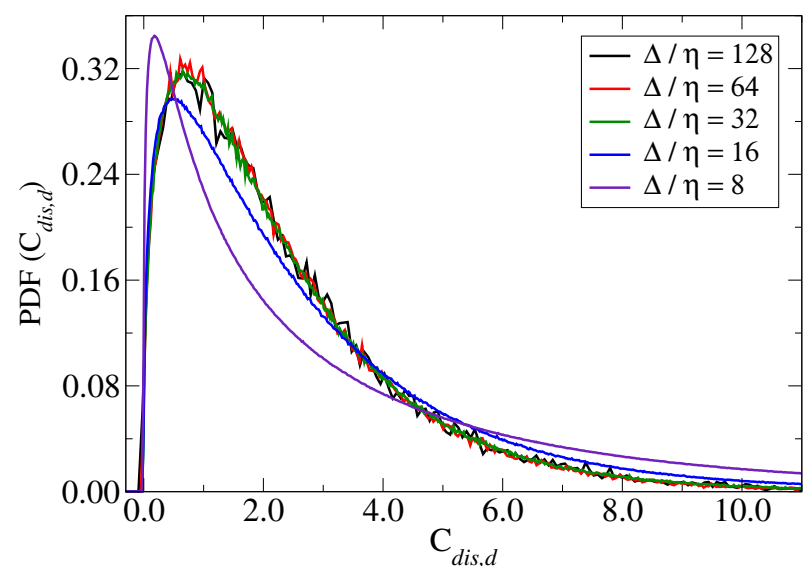

Figure 12: PDFs of the model coefficient $C_{d i s, d}$ from Eq. (26), evaluated from the homogeneous scalar mixing DNS. The PDFs are parameterized by the LES filter width, $\Delta$.

Note that the tails of the $C_{d i s, d}$ distribution are not associated with particularly high values of the filtered dissipation rate. Rather, plotting this model coefficient after conditioning it on the dissipation $\bar{\chi}_{Z}$ reveals that the tails of the PDF shorten when only large values of $\bar{\chi}_{Z}$ are considered.

The mean values of the $C_{d i s, d}$ distributions are approximately $C_{d i s, d}=2.3$, but the peaks of the distributions occur around $C_{d i s, d}=0.75$. Many RANS models use a dissipation model coefficient of approximately 1.0 [19] that sits in between these peak and mean values. To complete the closures for the LES $\widetilde{|\nabla Z|^{2}}$ equation the value $C_{d i s, d}=1.0$ is used, and the coefficient $C_{d i s}$ in Eq. (23) is modeled as

$$
C_{d i s}=C_{d i s, d} \cdot \frac{C_{v a r}}{C_{v a r}^{0}}=1.0 \cdot \frac{C_{v a r}}{(1 / 12)}=12 C_{v a r} .
$$


The LES transport equation for $\widetilde{|\nabla Z|^{2}}$ may then be written in final form as

$$
\begin{aligned}
\frac{D}{D t}\left(\bar{\rho} \mid \widetilde{\left.\nabla Z\right|^{2}}\right) & =\frac{\partial}{\partial x_{j}}\left(\bar{\rho}\left(\widetilde{\mathcal{D}}_{Z}+D_{t}\right) \frac{\partial}{\partial x_{j}}\left(\widetilde{|\nabla Z|^{2}}\right)\right) \\
& -2 \bar{\rho}\left(\frac{\partial \widetilde{u}_{i}}{\partial x_{j}} \frac{\partial \widetilde{Z}}{\partial x_{i}} \frac{\partial \widetilde{Z}}{\partial x_{j}}\right) \\
& -2 \bar{\rho} \widetilde{\mathcal{D}}_{Z}\left(\frac{\partial^{2} \widetilde{Z}}{\partial x_{i} \partial x_{j}}\right)^{2} \\
& -\frac{2}{\bar{\rho}} \frac{\partial \bar{\rho}}{\partial x_{i}} \frac{\partial \widetilde{Z}}{\partial x_{i}}\left(\frac{\partial}{\partial x_{j}}\left(\bar{\rho} \widetilde{\mathcal{D}}_{Z} \frac{\partial}{\partial x_{j}}(\widetilde{Z})\right)\right) \\
& +2 \frac{\partial\left(\bar{\rho} \widetilde{\mathcal{D}}_{Z}\right)}{\partial x_{i}} \frac{\partial \widetilde{Z}}{\partial x_{i}}\left(\frac{\partial^{2} \widetilde{Z}}{\partial x_{j} \partial x_{j}}\right) \\
& +2 \frac{\partial \widetilde{Z}}{\partial x_{i}} \frac{\partial \widetilde{Z}}{\partial x_{j}}\left(\frac{\partial^{2}\left(\bar{\rho} \widetilde{\mathcal{D}}_{Z}\right)}{\partial x_{i} \partial x_{j}}\right) \\
& +C_{p r d} \cdot \bar{\rho} \cdot \frac{32 \cdot \nu_{t}}{\Delta^{2}} \cdot\left(\widetilde{|\nabla Z|^{2}}-|\nabla \widetilde{Z}|^{2}\right) \\
& -12 \cdot C_{v a r} \cdot \bar{\rho} \cdot \frac{\widetilde{\mathcal{D}}_{Z}}{\widetilde{Z_{s f s}^{\prime \prime 2}}} \cdot\left(\widetilde{|\nabla Z|^{2}}-|\nabla \widetilde{Z}|^{2}\right)^{2}
\end{aligned}
$$

where $C_{p r d}=1.0$ and $C_{v a r}$ is the dynamically computed subfilter scalar variance coefficient from Eq. (5). The variance $\widetilde{Z_{s f s}^{\prime \prime 2}}$ that appears in the last term in Eq. (29) is determined from the solution of the conserved $\widetilde{Z}$ equation and the $\widetilde{Z^{2}}$ equation (Eq. (11)).

\section{Transport Equation Model Performance}

The transport equation models for $\bar{\chi}_{Z}$ and $\widetilde{Z_{s f s}^{\prime \prime 2}}$ are now evaluated by returning to the LES simulations of the reactive jet case. Additional computations of both the 1 million cell LES and the 23 million cell LES are performed, and the $\widetilde{|\nabla Z|^{2}}$ and $\widetilde{Z^{2}}$ variables are solved for using Eqs. (29) and (11), respectively. These two variables are used to locally compute the dissipation rate $\bar{\chi}_{Z}$ and the subfilter variance $\widetilde{Z_{s f s}^{\prime \prime 2}}$.

Contour plots of the LES transported dissipation rate and subfilter variance are shown in Fig. 13. Both the dissipation rate and the subfilter variance are seen to have increased relative to the algebraic model results in Fig. 3. Additionally, the high dissipation and high variance structures in the jet are seen to broaden somewhat when transport equation models are used. As quantified below, these changes bring the subfilter models into better agreement with the filtered DNS data.

Time averaged transported dissipation rates from both LES simulations are compared to the time averaged DNS dissipation rate and to the original algebraic model results in Fig. 14. The transport equation model improves upon the LES algebraic dissipation rate model in two ways. First, mesh dependencies are reduced. The algebraic model results were shown to be a strong function of the LES filter width. Conversely, the transport equation results in Fig. 14 are only weakly dependent on the LES filter. This change represents a significant improvement in model robustness. Second, the transported dissipation rate in the 1 million cell LES is in significantly better agreement with the DNS than the 1 million cell LES algebraic model. The improved agreement is attributed to two factors: 1) to 

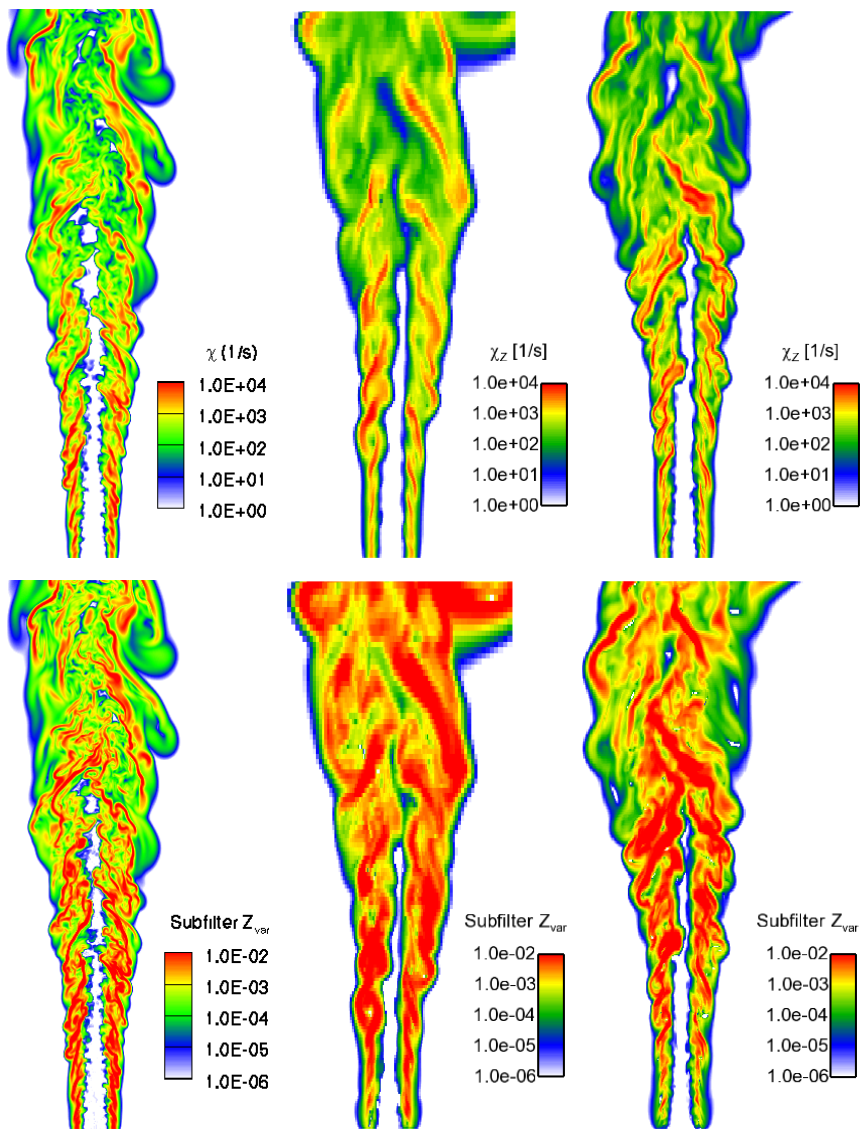

Figure 13: LES transport equation model results. Left column: DNS fields filtered using the 1 million cell LES filter width; Middle column: 1 million cell LES; Right column: 23 million cell LES. Top row: modeled $\bar{\chi}_{Z}$ from Eq. (29); Lower row: modeled $\widetilde{Z_{s f s}^{\prime \prime 2}}$ computed from Eq. (11) and the transported $\widetilde{Z}$ equation.

improved prediction of the subfilter variance production and variance dissipation processes that were inadequately represented by the algebraic approach, and 2) to the relaxation of the assumption that the dissipation rate is always in equilibrium with the local rate of variance production.

The time averaged subfilter variances that are obtained from the solution of the $\widetilde{Z^{2}}$ and $\widetilde{Z}$ equations are plotted in Fig. 15. This figure compares the transported LES variance with the corresponding DNS data and with the original algebraic variance model results. The use of a transport equation model has a pronounced effect on variance predictions, with predicted values increasing by an order of magnitude relative to algebraic model predictions. This increase results in the transport equation models significantly over-predicting subfilter variance at the $\mathrm{X} / \mathrm{H}=6$ and $\mathrm{X} / \mathrm{H}=7$ stations.

Several observations can be made regarding the transport equation model's over-prediction of subfilter variance. First, the model errors cannot be attributed to the closure of the dissipation term $\bar{\chi}_{Z}$ that appears in the $\widetilde{Z^{2}}$ equation, Eq. (11). Rather, Fig. 14 demonstrated that the transport equation model for this dissipation rate was accurate at both LES filter resolutions. Second, the variance transport equation model is much less robust to filter width changes than is the dissipation rate model. A comparison of the different filter res- 


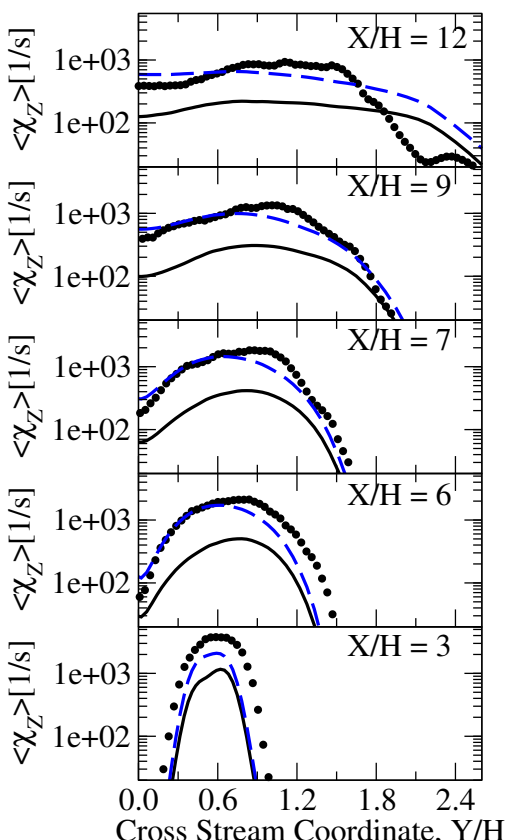

(a) 1 million cell LES filter width

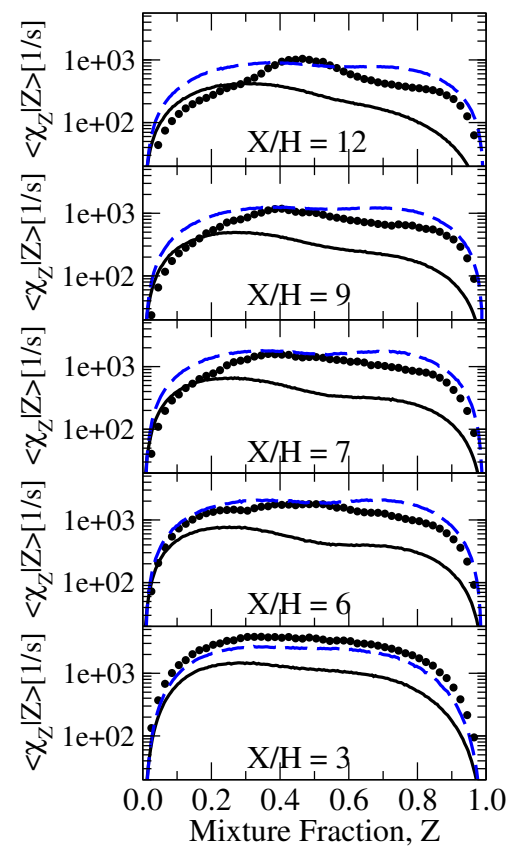

(c) 1 million cell LES filter width

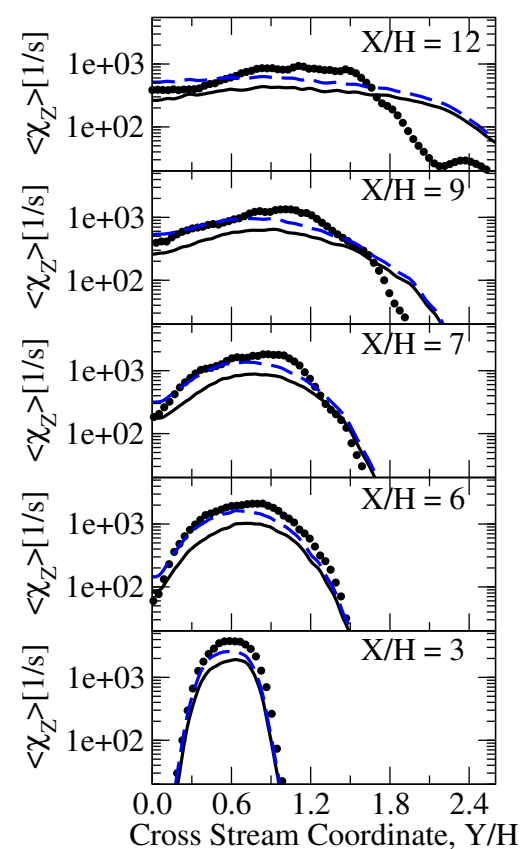

(b) 23 million cell LES filter width

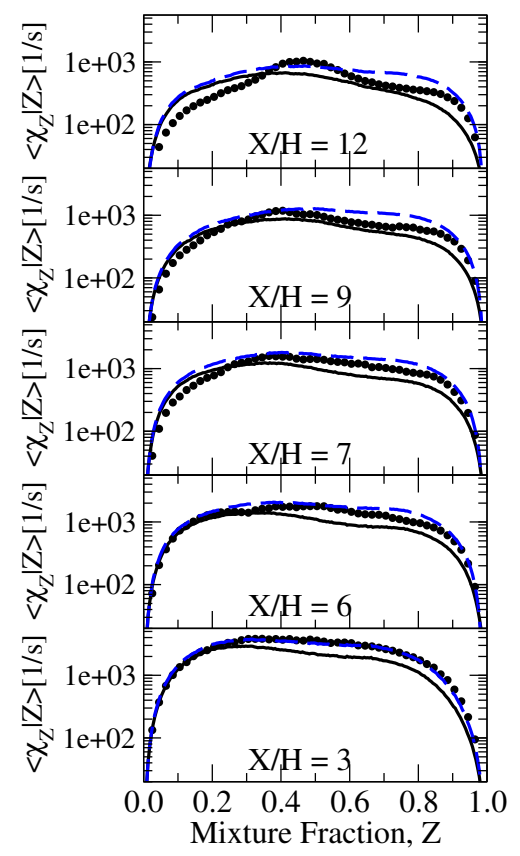

(d) 23 million cell LES filter width

Figure 14: Dissipation rate, $\bar{\chi}_{Z}$, in both physical and mixture-fraction conditioned space in the reacting jet.

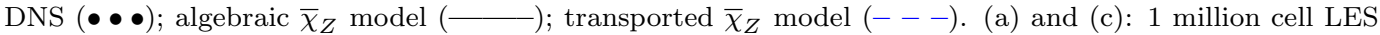
filter width; (b) and (d): 23 million cell LES filter width. 


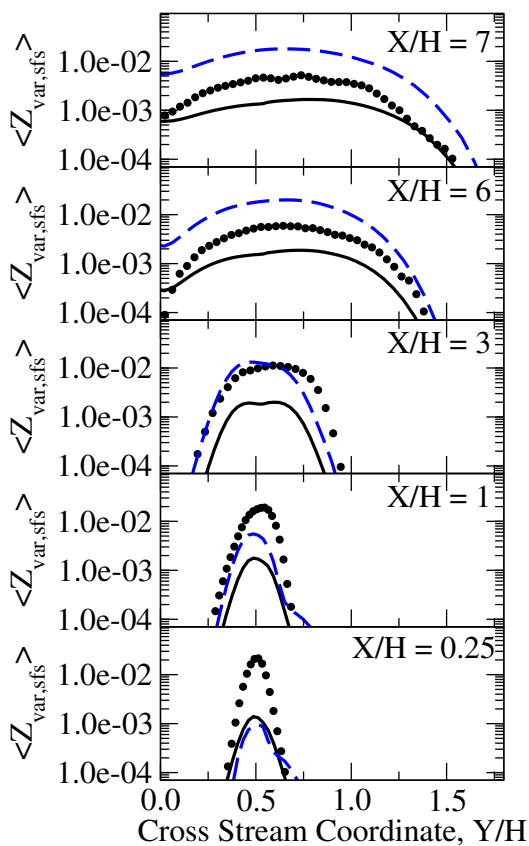

(a) 1 million cell LES filter width

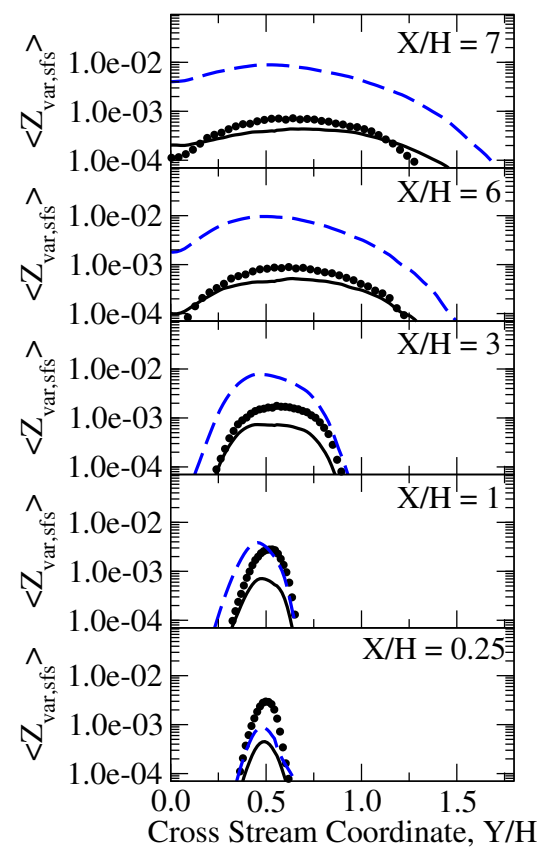

(b) 23 million cell LES filter width

Figure 15: Subfilter variance results, $\left\langle\widetilde{Z_{s f s}^{\prime \prime 2}}\right\rangle$, from the filtered DNS $(\bullet \bullet \bullet)$, the LES algebraic model $(-)$, and from the LES transport equation $(---)$. (a) 1 million cell LES filter width; (b): 23 million cell LES filter width.

olutions at the $\mathrm{X} / \mathrm{H}=7$ station in Fig. 15 shows that the maximum LES subfilter variance decreases only marginally with decreasing filter width, while the filtered DNS variance decreases significantly. Third, the relative accuracy of the algebraic and transport equation models is a function of the downstream jet coordinate. At locations near the jet inlet, both the transport equation and algebraic models strongly under-predict variance. At locations between $\mathrm{X} / \mathrm{H}=1$ and $\mathrm{X} / \mathrm{H}=3$, the transport equation model performs more accurately than the algebraic model. Finally, at locations farther downstream, this trend reverses and the algebraic model agrees most closely with the filtered DNS.

Errors in the transport equation model for the variance are most likely attributable to two sources. These sources are highlighted because, once Fig. 14 has been used to eliminate the dissipation term in Eq. (15) from consideration, they have the strongest influence on the transported variance model. The sources are 1) errors in the models for the subfilter convective flux terms in the $\widetilde{Z^{2}}$ and $\widetilde{Z}$ equations, and 2) numerical errors. These sources may be equivalently treated as 1) descriptions of subfilter turbulent mixing, and 2) descriptions of resolved turbulent mixing.

The subfilter flux models (source \#1) that are used in the LES are accurate enough to reasonably reproduce time averaged mixing profiles (Fig. 2), and to adequately capture the turbulent diffusivity in the reacting jet (Fig. 6). They nonetheless have difficulty modeling the detailed dynamics of the shear near the jet inlet (the $\mathrm{X} / \mathrm{H}=3$ station in Fig. 2), and it is well known that they cannot capture the tensorial nature of subfilter flux physics [37]. The importance of these flux model limitations is only expected to be magnified in the transported variance model, where differences between scalar solutions, rather than the magnitudes of single scalar quantities, are needed. 
Numerical errors (source \#2) are known to influence subfilter variance models through a variety of non-linear mechanisms [12] that include the over-prediction of gradients at the smallest resolved length scales of an LES, and the propagation of these over-predictions through dynamic modeling procedures. To estimate the importance of these influences in the reactive jet case, LES runs of both the 1 million and 23 million cell cases are performed using two different numerical schemes for the transport the $\widetilde{Z}, \widetilde{Z^{2}}$, and $\widetilde{|\nabla Z|^{2}}$ scalars. In the first set of runs, a third order QUICK scheme [38] is used to transport the scalars. In the second set of runs, a third order WENO scheme [39] is used for transport. The dissipation rates $\bar{\chi}_{Z}$ that are predicted using these different schemes are statistically identical, varying by no more than a few percent. The subfilter variances $\widetilde{Z_{s f s}^{\prime \prime 2}}$ that are predicted by the two schemes are shown in Fig. 16. Few differences are observed between the QUICK and WENO solutions in the 23 million cell LES, but the 1 million cell results respond strongly to numerics at downstream locations. In this coarse case, the slightly smoother scalar fields that result from the use of WENO lead to lower subfilter variance predictions that are in much better agreement with the filtered DNS.

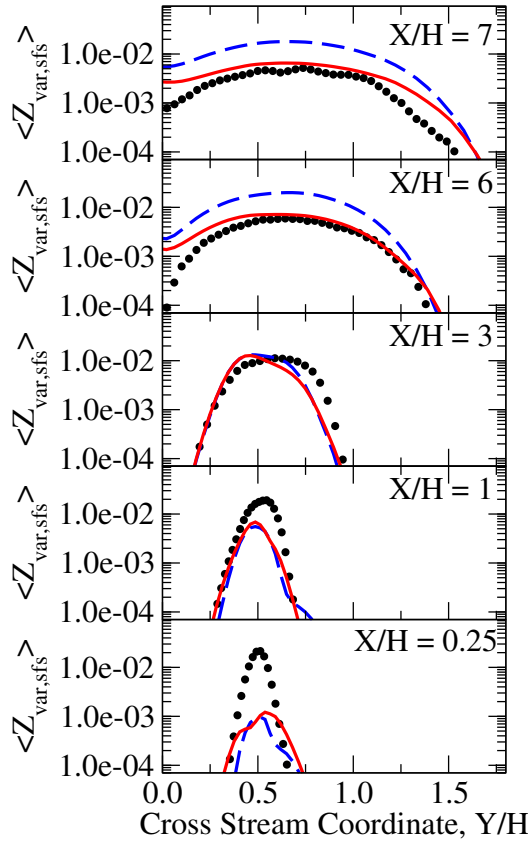

(a) 1 million cell LES filter width

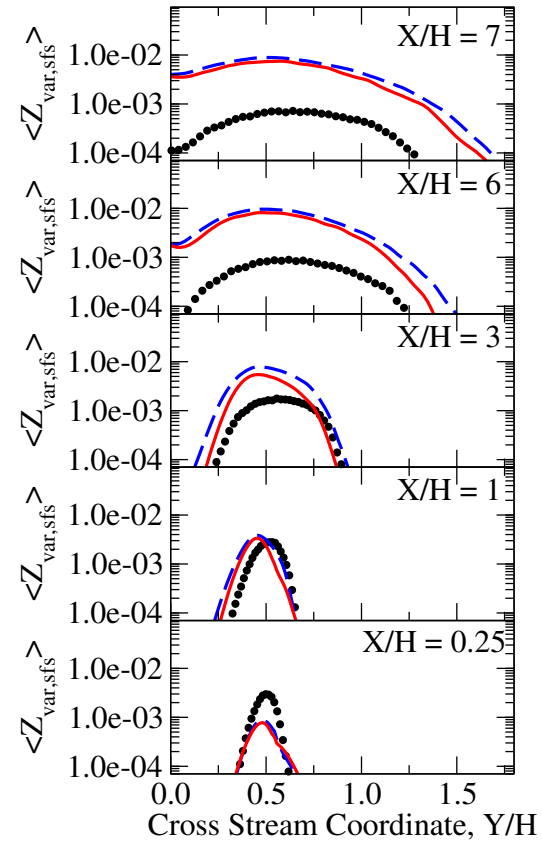

(b) 23 million cell LES filter width

Figure 16: Subfilter variance results, $\left\langle\widetilde{Z_{s f s}^{\prime \prime 2}}\right\rangle$, from the filtered DNS $(\bullet \bullet \bullet)$, the LES transport equation model solved using QUICK $(---)$, and from the LES transport equation model solved using WENO ( - ). (a) 1 million cell LES filter width; (b): 23 million cell LES filter width.

Although these numerical sensitivities provide context to the coarse LES predictions, they do not explain disagreements between the finer LES and the corresponding filtered DNS data. The errors in Fig. 16(b) may be caused by a more subtle numerical issue, or by a subfilter flux model's failure (source \#1 above) to reproduce the details of the turbulent mixing process at downstream locations. Fully addressing these issues will require further study. 


\section{Summary And Conclusions}

This work has used an auto-igniting jet DNS to analyze the performance of LES algebraic and transport equation models for both the scalar dissipation rate and the subfilter scalar variance. It was shown that the algebraic models under-predicted both the dissipation and the subfilter variance in the jet. The under-predictions were almost entirely due to the subfilter components of the algebraic expressions. These model errors motivated an examination of the closure of a transported scalar dissipation rate equation. An additional DNS of scalar mixing in homogeneous turbulence was introduced to aid this examination. The scalar mixing DNS demonstrated that subfilter production of $\bar{\chi}_{Z}$ could be described using a single model coefficient, while the coefficient associated with subfilter dissipation of $\bar{\chi}_{Z}$ was dependent on the LES filter. To account for this filter dependence, an adapted dynamic procedure was introduced and applied to the dissipation model coefficient. This procedure used information from a dynamic algebraic variance model to reduce the amount of physics that the dissipation model must describe. The resulting transport equation closure for $\bar{\chi}_{Z}$ was shown to perform well for a variety of LES filter widths. The transport equation models were then applied in the reacting jet LES. The transported $\bar{\chi}_{Z}$ model performed significantly better than the algebraic model and improved the agreement with the DNS results. The subfilter scalar variance predictions did not match the DNS data as accurately as did the dissipation predictions, but the dissipation rate that appears in the $\widetilde{Z^{2}}$ equation was eliminated from consideration as the source of these variance errors. Identifying the cause of the subfilter variance model errors requires further effort.

\section{Acknowledgements}

Support from the United States Air Force Office of Scientific Research (AFOSR), and from the National Aeronautics and Space Administration (NASA) is gratefully acknowledged.

[1] N. Peters, Turbulent Combustion, Cambridge Univ. Press, Cambridge, UK, 2000.

[2] N. Peters, Local quenching due to flame stretch and non-premixed turbulent combustion, Combust. Sci. Technol. 30 (1983) 1-17.

[3] C. D. Pierce, P. Moin, Progress-variable approach for large-eddy simulation of nonpremixed turbulent combustion, J. Fluid Mech. 504 (2004) 73-97.

[4] A. Y. Klimenko, R. W. Bilger, Conditional moment closure for turbulent combustion, Prog. Energy Comb. Sci. 25 (6) (1999) 595-687.

[5] G. D. Paola, E. Mastorakos, Y. M. Wright, K. Boulouchos, Diesel engine simulations with multi-dimensional conditional moment closure, Combust. Sci. Technol. 180 (2008) 883-899.

[6] S. B. Pope, PDF methods for turbulent reactive flows, Prog. Energy Combust. Sci. 11 (1985) 119.

[7] V. Eswaran, S. B. Pope, Direct numerical simulations of the turbulent mixing of a passive scalar, Phys. Fluids 31 (3) (1988) 506-520.

[8] C. D. Pierce, P. Moin, A dynamic model for subgrid-scale variance and dissipation rate of a conserved scalar, Phys. Fluids 10 (12) (1998) 3041-3044. 
[9] A. W. Cook, J. J. Riley, A subgrid model for equilibrium chemistry in turbulent flows, Phys. Fluids 6 (8) (1994) 2868-2870.

[10] D. Veynante, R. Knikker, Comparison between LES results and experimental data in reacting flows, J. Turbulence 7 (35) (2006) 1-20.

[11] G. Balarac, H. Pitsch, V. Raman, Development of a dynamic model for the subfilter scalar variance using the concept of optimal estimators, Phys. Fluids 20 (3) (2008) 1-9.

[12] C. M. Kaul, V. Raman, G. Balarac, H. Pitsch, Numerical errors in the computation of sub-filter scalar variance in large eddy simulations, Phys. Fluids 21 (5) (2009) 1-11.

[13] S. S. Girimaji, Y. Zhou, Analysis and modeling of subgrid scalar mixing using numerical data, Phys. Fluids 8 (5) (1996) 1224-1236.

[14] G. Balarac, H. Pitsch, V. Raman, Modeling of the sublter scalar dissipation rate using the concept of optimal estimators, Phys. Fluids 20 (091701) (2008) 1-4.

[15] C. M. Kaul, V. Raman, A posteriori analysis of numerical errors in sublter scalar variance modeling for large eddy simulations, Phys. Fluids 23 (035102) (2011) 1-14.

[16] P. Domingo, L. Vervisch, D. Veynante, Large-eddy simulation of a lifted methane jet flame in a vitiated coflow, Combust. Flame 152 (3) (2008) 415-432.

[17] G. R. Newman, B. E. Launder, J. L. Lumley, Modelling the behaviour of homogeneous scalar turbulence, J. Fluid Mech. 111 (1981) 217-232.

[18] W. P. Jones, P. Musonge, Closure of the Reynolds stress and scalar flux equations, Phys. Fluids 31 (12) (1988) 3589-3604.

[19] J. P. H. Sanders, I. Gökalp, Scalar dissipation rate modelling in variable density turbulent axisymmetric jets and diffusion ames, Phys. Fluids 10 (4) (1998) 938-948.

[20] M. Ihme, Y. C. See, Prediction of autoignition in a lifted methane/air flame using an unsteady flamelet/progress variable model, Combust. Flame 157 (2010) 1850-1862.

[21] H. Pitsch, L. Duchamp De Lageneste, Large-eddy simulation of premixed turbulent combustion using a level set approach, Proc. Comb. Inst. 29 (2002) 2001-2008.

[22] C. Meneveau, T. S. Lund, W. H. Cabot, A Lagrangian dynamic subgrid-scale model of turbulence, J. Fluid Mech. 319 (1996) 353-385.

[23] C. Wall, B. J. Boersma, P. Moin, Evaluation of the assumed beta probability density function subgrid-scale model for large eddy simulation of nonpremixed, turbulent combustion with heat release, Phys. Fluids 12 (10) (2000) 2522-2529.

[24] C. S. Yoo, R. Sankaran, J. H. Chen, A DNS study on the stabilization mechanism of a turbulent lifted ethylene jet flame in highly-heated coflow, Proc. Comb. Inst. 33 (2011) $1619-1627$.

[25] T. Passot, A. Pouquet, Numerical-simulation of compressible homogeneous flow in the turbulent regime, J. Fluid Mech. 181 (1) (1987) 441-466.

[26] H. Pitsch, Unsteady flamelet modeling of differential diffusion in turbulent jet diffusion flames, Combust. Flame 123 (2000) 358-374.

[27] H. Pitsch, N. Peters, A consistent flamelet formulation for non-premixed combustion considering differential diffusion effects, Combust. Flame 114 (1998) 26-40. 
[28] L. Vervisch, P. Domingo, G. Lodato, D. Veynante, Scalar energy fluctuations in largeeddy simulation of turbulent flames: Statistical budgets and mesh quality criterion, Combust. Flame 157 (4) (2010) 778-789.

[29] O. Desjardins, G. Blanquart, G. Balarac, H. Pitsch, High order conservative finite difference scheme for variable density low Mach number turbulent flows, J. Comput. Phys. 227 (15) (2008) 7125-7159.

[30] C. A. Kennedy, J. H. Chen, Mean flow effects on the linear stability of compressible planar jets, Phys. Fluids 10 (3) (1998) 615-626.

[31] P. Vedula, P. K. Yeung, R. O. Fox, Dynamics of scalar dissipation in isotropic turbulence: a numerical and modelling study, J. Fluid Mech. 433 (35) (2001) 29-60.

[32] J. W. Deardorff, Stratocumulus-capped mixed layers derived from a 3-dimensional model, Boundary-Layer Meteorology 18 (4) (1980) 495-527.

[33] E. M. Doran, A multi-dimensional flamelet model for ignition in multi-feed combustion systems, Ph.D. thesis, Stanford University (2011).

[34] C. Rosales, C. Meneveau, Linear forcing in numerical simulations of isotropic turbulence: physical space implementations and convergence properties, Phys. Fluids 17 (9) (2005) 1-8.

[35] E. M. Doran, H. Pitsch, A bivariate beta distribution as a presumed pdf for multiple scalar mixing, Submitted to Phys. Fluids.

[36] E. Knudsen, S. H. Kim, H. Pitsch, An analysis of premixed flamelet models for large eddy simulation of turbulent combustion, Phys. Fluids 22 (115109) (2010) 1-24.

[37] C. Meneveau, J. Katz, Scale-invariance and turbulence models for large-eddy simulation, Ann. Rev. Fluid Mech. 32 (2000) 1-32.

[38] B. P. Leonard, A stable and accurate convective modelling procedure based on quadratic upstream interpolation, Comput. Methods Appl. Mech. Eng. 19 (1979) 59-98.

[39] G.-S. Jiang, D. Peng, Weighted ENO schemes for Hamilton-Jacobi equations, J. Sci. Comput. 21 (6) (2000) 2126-2143. 